NASA/TM-2018-219752

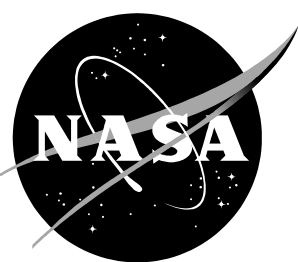

Vortex Shedding Characteristics of the Wake of a Thin Flat Plate with a Circular Trailing Edge

Man Mohan Rai

Ames Research Center

Moffett Field, CA-94035 


\section{NASA STI Program ... in Profile}

Since its founding, NASA has been dedicated to the advancement of aeronautics and space science. The NASA scientific and technical information (STI) program plays a key part in helping NASA maintain this important role.

The NASA STI program operates under the auspices of the Agency Chief Information Officer. It collects, organizes, provides for archiving, and disseminates NASA's STI. The NASA STI program provides access to the NTRS Registered and its public interface, the NASA Technical Reports Server, thus providing one of the largest collections of aeronautical and space science STI in the world. Results are published in both nonNASA channels and by NASA in the NASA STI Report Series, which includes the following report types:

- TECHNICAL PUBLICATION. Reports of completed research or a major significant phase of research that present the results of NASA Programs and include extensive data or theoretical analysis. Includes compilations of significant scientific and technical data and information deemed to be of continuing reference value. NASA counterpart of peer-reviewed formal professional papers but has less stringent limitations on manuscript length and extent of graphic presentations.

- TECHNICAL MEMORANDUM.

Scientific and technical findings that are preliminary or of specialized interest, e.g., quick release reports, working papers, and bibliographies that contain minimal annotation. Does not contain extensive analysis.

- CONTRACTOR REPORT. Scientific and technical findings by NASA-sponsored contractors and grantees.
- CONFERENCE PUBLICATION.

Collected papers from scientific and technical conferences, symposia, seminars, or other meetings sponsored or co-sponsored by NASA.

- SPECIAL PUBLICATION. Scientific, technical, or historical information from NASA programs, projects, and missions, often concerned with subjects having substantial public interest.

- TECHNICAL TRANSLATION. English-language translations of foreign scientific and technical material pertinent to NASA's mission.

Specialized services also include organizing and publishing research results, distributing specialized research announcements and feeds, providing information desk and personal search support, and enabling data exchange services.

For more information about the NASA STI program, see the following:

- Access the NASA STI program home page at http://www.sti.nasa.gov

- E-mail your question to help@sti.nasa.gov

- Phone the NASA STI Information Desk at 757-864-9658

- Write to: NASA STI Information Desk

Mail Stop 148

NASA Langley Research Center Hampton, VA 23681-2199 
NASA/TM-2018-219752

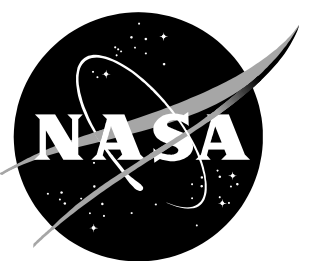

Vortex Shedding Characteristics of the Wake of a Thin Flat Plate with a Circular Trailing Edge

Man Mohan Rai

Ames Research Center

Moffett Field, California

National Aeronautics and

Space Administration

Ames Research Center

Moffett Field, California 94035-1000

January 2018 


\title{
VORTEX SHEDDING CHARACTERISTICS OF THE WAKE OF A THIN FLAT PLATE WITH A CIRCULAR TRAILING EDGE
}

\author{
Man Mohan Rai \\ Senior Researcher, Computational Sciences, Exploration Technology Directorate \\ NASA Ames Research Center, Moffett Field, CA-94035, USA
}

\begin{abstract}
The near and very near wake of a thin flat plate with a circular trailing edge are investigated with direct numerical simulations (DNS). Data obtained for two different Reynolds numbers (based on plate thickness, D) are the main focus of this study. The separating boundary layers are turbulent in both cases. An earlier investigation of one of the cases (Case F) showed shed vortices in the wake that were about 1.0D to 4.0D in spanwise length. Considerable variation in both the strength and frequency of these shed vortices was observed. One objective of the present investigation is to determine the important contributors to this variability in strength and frequency of shed vortices and their finite spanwise extent. Analysis of the data shows that streamwise vortices in the separating boundary layer play an important role in strengthening/weakening of the shed vortices and that high/low-speed streaks in the boundary layer are important contributors to variability in shedding frequency. Both these features of the boundary layer contribute to the finite extent of the vortices in the spanwise direction. The second plate DNS (Case G, with $40 \%$ of the plate thickness of Case F) shows that while shedding intensity is weaker than obtained in Case $F$, many of the wake features are similar to that of Case $F$. This is important in understanding the path to the wake of the thin plate with a sharp trailing edge where shedding is absent. Here we also test the efficacy of a functional relationship between the shedding frequency and the Reynolds numbers based on the boundary layer momentum thickness $\left(R_{e}\right)$ and $D\left(R e_{D}\right)$; data for developing this behavioral model is from Cases $F \& G$ and five earlier DNSs of the flat plate wake.
\end{abstract}

\section{INTRODUCTION}

The wake of the thin flat plate with a sharp trailing edge and turbulent boundary layers has been discussed in several articles, one of the earliest being that of Chevray \& Kovasznay (1969). The ratio of the boundary layer momentum thickness to the trailing edge thickness of the plate ( $\theta / D)$ is large (23.2) in their study. Profiles of measured mean velocity and turbulent normal intensities and shear stress are provided. The boundary layers merge gradually to form the wake. Large-scale vortex shedding is absent. Other notable experimental investigations of the thin flat plate with a sharp trailing edge include those of Ramaprian et al (1982), Nakayama \& Liu (1990), Hayakawa \& lida (1992) and Thomas \& Liu (2004). In addition to the experimental investigations mentioned above analytical solutions based on certain simplifying assumptions are provided by Alber (1980). In this study the centerline velocity distribution in the $x$ direction in a region of the near wake is approximated by a logarithmic relation similar in form to that obtained for the turbulent boundary layer upstream of the wake in these cases. A good comparison is obtained between experimental data and the 'wake log-law' in the near wake. A more detailed review of these articles is provided in Rai (2017).

In contrast to the thin plate with a sharp trailing edge, the thick plate with a blunt trailing edge (small $\theta / D$ ) exhibits vigorous vortex shedding. Unlike the case of the cylinder, the Reynolds number based on momentum thickness of the boundary layer just upstream of the trailing edge $\left(R_{\theta}\right)$ and the Reynolds number defined using the thickness of the flat plate or the diameter of its trailing edge $\left(\operatorname{Re}_{\mathrm{D}}\right)$, are 
independent parameters. A detailed investigation of the wake of the thick flat plate with a circular trailing edge and turbulent separating boundary layers, was initiated by Rai $(2013,2014$ \& 2015). This was accomplished with the aid of direct numerical simulations (DNS). The boundary layers as well as the wake were computed via DNS in these investigations. The separating boundary layers are fully turbulent well upstream of the trailing edge and are statistically identical. Thus the wake is symmetric in the mean.

Collectively, the articles by Rai $(2013,2014$ \& 2015) address several aspects of flat plate wake flow. The more important ones are noted here. The distributions of phase-averaged turbulent intensity and shear stress (random component) in the near and very near wakes are provided; wherever possible a physical explanation of the origin of the important features of the distributions, as well as one based on the distribution of the production term in the corresponding budget, are provided. A new event-based phase-averaging procedure is introduced. Important flow features such as rib vortices and their structure and strength in relation to the shed vortices and evolution in time and the internal structure of braids \& shed vortices are investigated. A study of the relative importance of phase-averaged and fluctuating strain rates in the production of turbulent vorticity in the braids and cores showed that the former is less important than the latter in generating turbulent vorticity.

Similar to the shear-layer instability that manifests in the case of the cylinder with laminar separating boundary layers it was found that the flat plate wake also exhibits shear layer instability followed by the formation of shear layer vortices that have a profound impact on the structure of the shear layer and the formation of the shed vortices. However, unlike the cylinder cases, here only a small fraction of the separated turbulent boundary layer participates in the initial formation of the shed vortices, and it is this fraction that is unstable. The role of recirculation region vortices and log-layer eddies in generating the shear-layer vortices associated with this instability was investigated and established; both sets of vortices play a role in initiating this instability. A visualization of shear-layer instability events in a ( $t, z)$ plane showed that shear-layer vortex generation rates can vary as much as a factor of two from event to event. An analysis of velocity fluctuations in the upstream boundary layer indicated that high-speed streaks near the trailing edge result in higher shear-layer vortex generation rates.

The existence of regions of isolated reverse flow that are disconnected from the main body of reverse flow in the trailing edge region was discovered in Rai (2014). They are first formed near the trailing edge and convect downstream. These regions are a result of powerful rib vortices that are formed in the high-strain-rate region that exists between the shed vortices in their initial state; these regions are quite energetic with streamwise velocities (negative) within them reaching $40 \%$ of the freestream velocity. A spectral analysis of the $z$-averaged negative streamwise velocity showed a peak at twice the vortex shedding frequency. This correspondence between the two frequencies is a result of the important role that shed vortices play in the generation of these regions of reverse flow.

The role that entrainment plays in the case of the near wake of a thick flat plate is quite different than it does in the case of the far wake. As mentioned earlier, only a small fraction of the separating turbulent boundary layer forms the detached shear layer (DSL) and participates in the initial roll-up into the shed vortex. The log-layer eddies of the boundary layer travel past the trailing edge largely unaltered. A detailed investigation of the entrainment process was performed. It was determined that for some distance downstream, the wake with its shed vortices, ingests fluid that was originally part of the turbulent boundary layer. The log-layer eddies are assimilated in this process and become a part of the shed vortices or the braids; a visualization is provided in Rai (2015). The effect of increasing $\theta / D$ on assimilation/entrainment was also investigated in this article; it showed that wakes with larger $\theta / D$ values continue to assimilate boundary layer fluid for longer (until a larger value of $x / D$ ); the important contributors to this effect are identified. As a consequence of this gradual assimilation process, wake TKE profiles in the direction normal to the wake, in the region away from the wake-core containing the shed 
vortices and braids, are close to that of the upstream turbulent boundary layer; especially in the very near wake for the large $\theta / D$ cases.

The objective of the more recent investigation by Rai (2017) is to better understand the changes in the characteristics of the wake of a flat plate with turbulent separating boundary layers (in particular changes in shedding and related wake features), as the plate becomes thin in relation to the boundary layer thickness (increasing $\theta / D$ ). The trailing edge of the plate remains circular in all the cases investigated in Rai (2017). Thus the wake flow is different from those obtained in the experimental investigations with sharp trailing edges that were mentioned earlier. Changes obtained with increasing $\theta / D$ in the coherence of the shed vortices (in the spanwise direction), roll-up of the detached shear layers, the basic shedding mechanism (initiation of circulation), substantial variation in shedding frequency for the case with the largest value of $\theta / D$, centerline velocity spectra and time-averaged velocity statistics are discussed in this article. Some of the reasons underlying the substantial changes in the observed flow features as $\theta / D$ is increased are provided. The data used in the investigation were obtained from direct numerical simulations of flat plate flow for four different values of $\mathrm{Re}_{\mathrm{D}}$ (at constant plate length). The turbulent boundary layers and the wake are all computed via DNS.

The four cases investigated in Rai (2017) are labeled Cases A, D, E \& F. Case A is considered as the reference case. Values of $\operatorname{Re}_{\mathrm{D}}$ and the ratio $\psi=(\theta / D) /(\theta / D)_{\text {Case } A}$ for the different cases are provided in Table 1. The ratio $\psi$ is essential in comparing the value of $(\theta / D)$ for any given case to that of the reference case (Case A); it varies substantially over the cases considered (by a factor of 20.43). Table 1 also provides the value of $\psi$ for the case computed during the present investigation, Case G. The Reynolds number based on plate length $L$ is the same in all cases, $R_{L}=1.25 \times 10^{6}$.

\begin{tabular}{|c|c|c|}
\hline & $\mathrm{Re}_{\mathrm{D}}$ & $\psi$ \\
\hline Case A & 10,000 & 1.00 \\
\hline Case D & 5000 & 2.28 \\
\hline Case E & 2500 & 4.89 \\
\hline Case F & 625 & 20.43 \\
\hline Case G & 250 & 51.59 \\
\hline
\end{tabular}

Table 1. The parameters $\operatorname{Re}_{D}$ and $\psi$ for Cases A, D, E, F (Rai 2017) and G.

One of the findings of Rai (2017) is the loss of coherence in the spanwise structure of the shed vortices as $\theta / D$ is increased. Figure 1 (Fig. 6 in Rai 2017) shows a top view of instantaneous surfaces of constant pressure for Cases $A, D \& E$ in the wake; $x$ and $z$ are the streamwise and spanwise directions, respectively. Figure 1a shows an instantaneous constant pressure surface for Case $A$. The shed vortices are clearly observed as nearly cylindrical regions of low pressure. They show little distortion in the $z$ direction. Some of the rib vortices in the braid regions connecting consecutive shed vortices of opposite sign are also evident as narrow tubes; a few are highly distorted. Shed vortices that are ruptured or that show large distortions are rare in Case A. Figure 1b shows an instantaneous constant pressure surface for Case D. The larger spanwise distortions near the base region (in comparison to Case A) and what appears to be the beginning of vortex breakdown further downstream is evident (less structured shedding as $\theta / D$ increases by a factor of 2.28). Because it is tempting to consider this loss in coherency as solely due to vortex breakdown processes, we hasten to add that the complexity in vortex structure is sometimes evident at shed vortex inception very near the trailing edge. This indicates that, at times, the formation process itself varies considerably from the norm. Case $E$ (Fig. 1c, which involves a further increase of $\theta / D$ ) yields significant vortex distortion in the entire near-wake region. It is clear from Figs. 1a $-1 \mathrm{c}$ that increasing $\theta / \mathrm{D}$ beyond a certain level results in the loss of coherence of shed vortices. It should be noted that although the spanwise extent of the computational region in Cases $D$ \& $E$ in Fig. 1 is $4.0 D$ and 8.0D, respectively, the extent in the $z$ direction in the two cases in relation to the plate length $L$ is the same ( $L$ is a constant in all four cases). 
Contours of instantaneous spanwise vorticity in a ( $\mathrm{x}, \mathrm{y})$ plane for Cases $\mathrm{A}$ and $\mathrm{E}$ are compared in Rai (2017) to better understand the loss in coherence with increasing $9 / \mathrm{D}$. As mentioned in Rai (2014), the shed vortex at inception in Case $A$ is not a single cylindrical vortex as in low Reynolds number cylinder flows. Instead, it is an amalgam of several smaller vortices of both signs. Vortices of the same sign as the vorticity in the shear layer that is rolling up are dominant. In addition, segments of the shear layer and shear-layer vortices are incorporated in the newly forming vortex. The comparison in Rai (2017) showed that the differences between the two cases are striking. Firstly, relative to the thickness of the plate, the thickness of the DSL in Case $E$ is considerably larger than in Case A. Secondly, much of the small-scale activity seen in Case A is absent in Case E. The third and perhaps the most important difference is that the two detached shear layers (DSLs) in Case E are separated by a physical distance that is approximately one quarter of that in Case $A$ (the trailing edge diameter in Case $E$ is 0.25 times that in Case A). The smaller separation results in a stronger interaction between the DSLs in Case $E$. There is less room for a more conventional, approximately circular, roll-up of the DSLs as in Case A. Instead, often a folding of the DSL into two distinct layers occurs in Case E. The resulting shed vortex is initially stretched out in the $x$ direction and sometimes breaks up into multiple shed vortices that occasionally reconstitute into a single larger shed vortex. The end result is the lack of coherence seen in Fig. 1c.

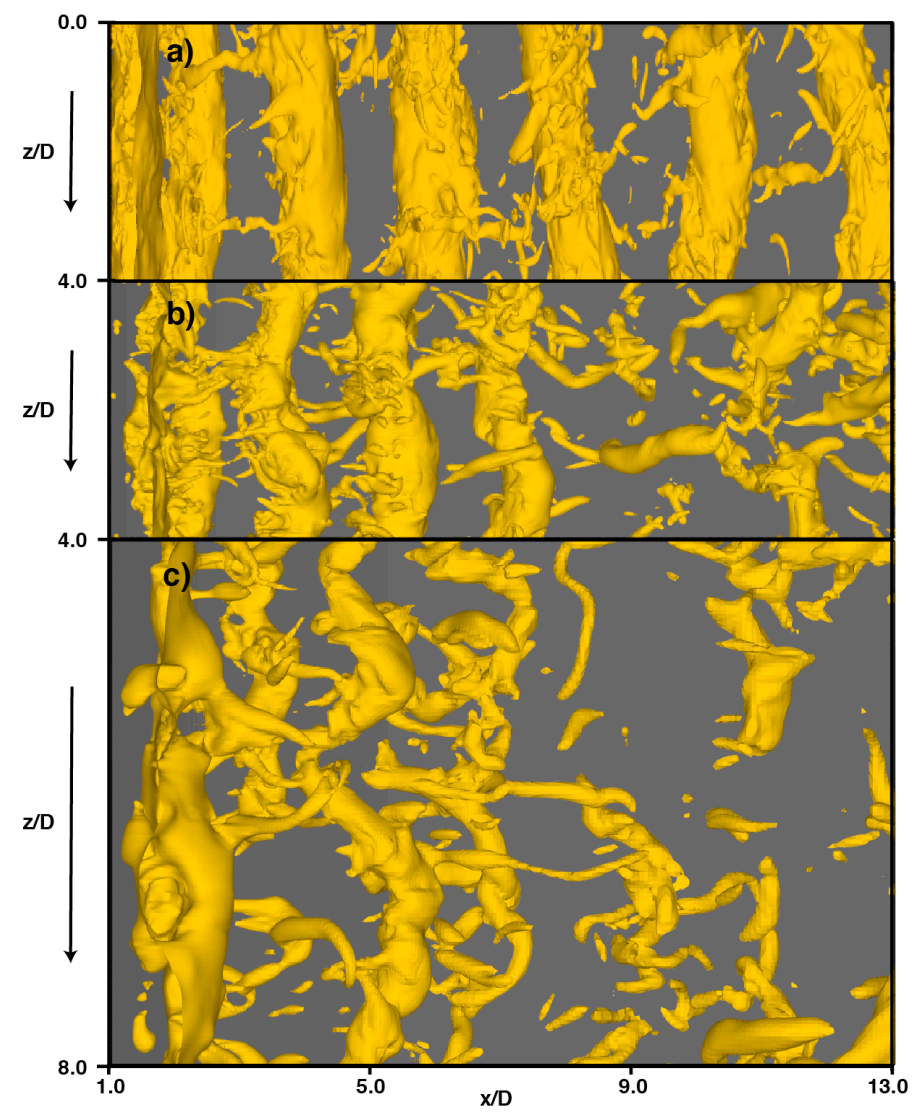

Figure 1. Surfaces of instantaneous constant pressure (top-view), a) Case $\mathrm{A}(\psi=1.00, \mathrm{n}=$ $70000)$, b) Case $D(\psi=2.28, n=180,000)$, c) Case $E(\psi=4.89, n=192,000)$; Figs. 6a, $6 b$ \& 6 c in Rai (2017).

The increases in $\theta / D$ in cases $D$ and $E$ in comparison to Case $A$ (increase in $\psi$ ) resulted in the initiation of deterioration in the shedding process. In Case F, where $\psi$ is large (20.43), the observed shedding characteristics are significantly different from the earlier cases. The spanwise extent of the computational domain in Case $F$ normalized by $L$ is the same as in Cases $D \& E$ (but in terms of the 
diameter it is 32.0D). A top view of instantaneous surfaces of constant negative $\lambda_{2}\left(\lambda_{2}\right.$ is the second eigenvalue of $S^{2}+\Omega^{2}$, see Jeong \& Hussain, 1985) in the region $-1.0<y / D<1.0$, for Case $F$ are provided in Fig. 2. While surfaces of constant pressure also show similar features, surfaces of constant negative $\lambda_{2}$ more clearly depict the vortices in Case $F$. The vorticity field in this case is very different from that obtained in Cases A, D \& E. Sequences of approximately spanwise vortices, between 1.0D \& 4.0D in length, are evident. Some of the sequences are marked with arrows. While two of the marked sequences show vortices well aligned with the $z$ direction, one occurs at a significant angle to this direction. Some of the vortices are approximately aligned with the $x$ direction. In the trailing edge region many of them are nearly streamwise vortices from the upstream boundary layers. The two most significant features of Fig. 2 are a) the spanwise vortices are of finite length in the $z$ direction and $b$ ) the sequences in time are finite. If indeed the sequences comprise shed vortices, then shedding seems to be intermittent in Case F.

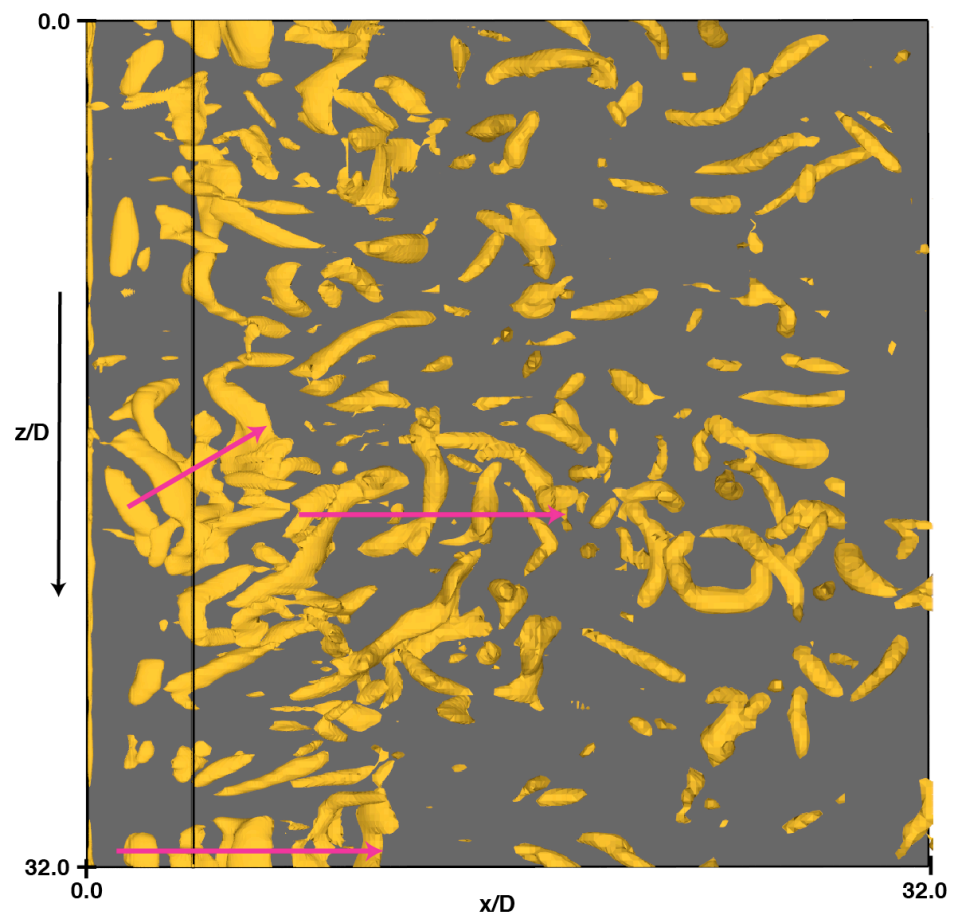

Fig. 2. Surfaces of instantaneous constant $\lambda_{2}$ (negative) in the region $-1.0<y / D<1.0$, Case $F$, (top view, $\psi=20.43, \mathrm{n}=200,000$ ); Fig. 13 in Rai (2017).

Some of the questions that Fig. 2 raises are as follows: Is the shedding process similar to the more conventional shedding of Cases A \& D? What is the cause of the observed intermittency in shedding? What causes the shed vortices to be of finite length? How does the spectrum obtained for the cross-stream velocity in Case $F$ compare with that of Case $D$ at the wake center-plane? Does the spectrum have a sharp peak? How do the fluctuations in velocity compare in the two cases? As demonstrated in Rai (2017), Case F does exhibit continuous vortex shedding although the shed vortices during certain periods in time are very weak.

We now summarize some of the findings of Rai (2017) before discussing the objectives of the present investigation. The DNSs of the flat plate wakes show that vortex shedding is vigorous in the low $\theta / D$ cases (Cases $A$ and $D$ ) and that there is a substantial decrease in shedding intensity as $\theta / D$ increases (Cases E \& F). A lack of coherence in the spanwise structure of the shed vortices is observed with increasing $\theta / D$. The interaction between the upper and lower shear layers becomes stronger as $\theta / D$ is increased. The proximity of the shear layers in the large $\theta / D$ cases, essentially reduces the ability of the shear layer to roll-up and contribute to the strength of the newly formed shed vortices. In Case $F$ the shear layers are so close to each other that the roll-up is essentially prevented and the shed vortices form 
and remain within the shear layer until the shear layer breaks up between 15 and 20 diameters from the trailing edge. Although a conventional roll-up is largely prevented in Case F, the shed vortices because of their combined induced velocity field, result in shear layers that are sinuous in appearance.

An initial examination of shedding in Case $\mathrm{F}$ via contours of cross-stream velocity at the wake center-plane $(t-z)$ plane) at a given $x$ location and also the method of Jeong \& Hussain $\left(1985, \lambda_{2}\right.$ criterion) indicated that vortex shedding may be intermittent. Quiescent regions, between regions of shedding activity, both in time and in the $z$ direction are observed. The former are an indication of potential intermittency in the shedding process. However, a closer look at the shedding process, using fluctuations in trailing-edge surface vorticity (and animations of velocity vectors), shows that vortex shedding is continuous in time although greatly weakened during certain periods. The active/quiescent regions may be a result of boundary layer disturbances that aid/inhibit vortex shedding. The role of boundary layer velocity fluctuations near the trailing edge as a causative agent of noticeably large fluctuations in shedding period was examined in brief in Rai (2017); the potential for high- and low-speed streaks to cause decreases/increases in shedding period, respectively, was noted.

Velocity vector and pressure contour plots were used in the case of the flat plate to understand the initiation of the shedding process in Cases A \& F (lowest and highest $\theta / D$ cases computed) and compare it to that of the cylinder at low Reynolds numbers in Rai (2017). The essential attributes of the initiation process in these two flat plate cases are quite similar to that found in the cylinder wake. The induced velocity fields of the previous two shed vortices and the forward flow in the detached shear layer and further away from the centerline result in the initiation of circulating flow very near the base in Cases $A$ \& $F$ (as in the case of the cylinder). Both shear-layer roll-up and the pressure minimum (associated with the shed vortex) only appear later in the process in Cases A, F and the cylinder.

The effect of changing the ratio $\theta / D$ on the time-averaged, near-wake velocity statistics was also investigated in Rai (2017, Cases D \& F). It was found that increasing $\theta / D$ resulted in weakened reverse flow in the base region that persists for longer distances (in terms of the thickness of the plate). Contours of time-averaged spanwise vorticity showed that increasing $\theta / D$ resulted in decreasing the separation between the upper and lower detached shear layers and increasing their streamwise extent. Peak crossstream intensity along the wake centerline is substantially lowered with increasing $\theta / D$ because of weaker shed vortices. The peak streamwise intensity is also lowered but by a much smaller factor because of the turbulent nature of the separated boundary layer (and thus the inherited streamwise intensity). Interestingly, distributions of the components of velocity, fluctuating kinetic energy and shear stress are found to remain topologically quite similar with changing $\theta / D$. However, the scales and locations of the various features such as maxima/minima are considerably different.

Here we continue the analysis of the wake of the thin flat plate initiated in Rai (2017); the data used is primarily from Case $F$ (computed in the earlier investigation) and Case $G$ that was computed as a part of current study (plate thickness is $40 \%$ of that in Case F). One objective of the present investigation is to determine the important contributors to the variability in the strength and frequency of shed vortices and their finite extent in the spanwise direction. The second objective is to understand the effect of a continued increase in $\theta / D$ on shedding characteristics. This is important in understanding the path to the wake of the thin plate with a sharp trailing edge where shedding is absent. Case $G$ was mainly computed for this purpose. In addition, a functional relationship between the shedding frequency and $\operatorname{Re}_{\theta} \& \mathrm{Re}_{\mathrm{D}}$ is tested; data for developing this behavioral model is from Cases A, D, E, F and two earlier DNSs (Cases $B$ \& $C$ from Rai 2015) of the flat plate. The shedding frequency predicted by the model for Case $G$ is compared with the value computed via DNS to determine the model's efficacy. Here the model is used in an extrapolative and not an interpolative mode. The exercise also establishes the relative importance of the two independent Reynolds numbers $R_{\theta} \& R_{D}$ in determining shedding frequency (as indicated by the model and its constants). The discussion is provided in an Appendix since it involves both thick and thin flat plates (small and large $\theta / D$ cases). 


\section{FLAT-PLATE COMPUTATIONAL GRID, FLOW/GEOMETRY PARAMETERS AND NUMERICAL METHOD}

The computational region for the flat plate DNSs is divided into two zones to facilitate grid generation and provide adequate grid resolution for the wake. Figure 3 shows the plate cross-section and the two zones that comprise the computational region. The three-dimensional zones and grids are obtained by uniformly spacing copies of these two-dimensional zones in the spanwise direction $(z)$. The plate zone is bounded by four boundaries: the plate surface (excluding the trailing edge), an external boundary and, two zonal boundaries (top and bottom) that interface with the wake zone. The wake zone is constructed to provide adequate grid resolution for the detached shear layers, the recirculation region and the wake. The boundaries of this zone include the circular trailing edge, the upper and lower boundaries and the exit boundary. Both the upper and lower boundaries consist of a zonal boundary segment that interfaces with the plate zone and a second segment that serves as an external boundary. Direct numerical simulations for five different values of $\mathrm{Re}_{\mathrm{D}}$ were performed to obtain the data of direct interest in this study (Cases A, D, E, F \& G). Of these, only Case G was computed as a part of the current investigation. The coordinate system shown in Fig. 3 is only indicative of the coordinate directions; the origin is at the center of the circular trailing edge (as in Fig. 4).

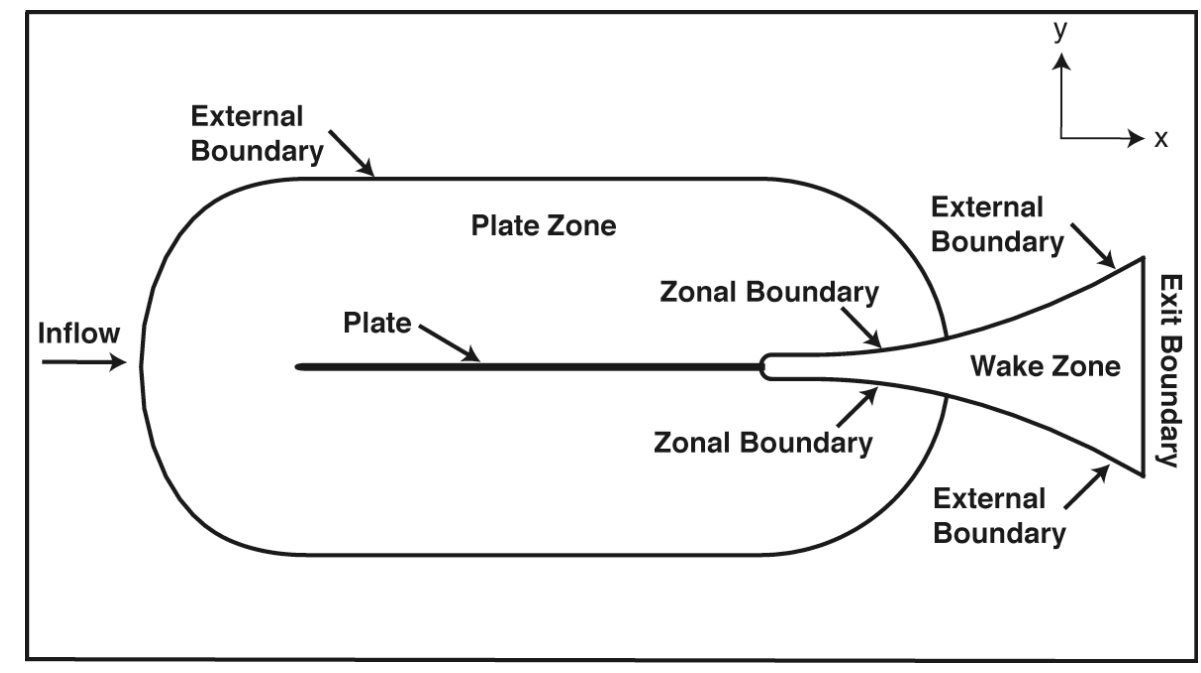

Figure 3. Midspan plate section and multiple zone discretization of the computational region (Rai 2013).

The placement of the various boundaries in relation to the plate surface in Cases $A \& D$ is provided in Rai $(2013,2014$ \& 2015). The vertical extent of the wake zone near the trailing edge, where its upper/lower boundaries are horizontal is large enough to completely contain the wake in all cases (Fig. 4 in Rai 2013 clearly shows the adequacy of this dimension in Case A). The spanwise extent of the region in Cases A \& D is 4.0D. Cases A \& D of the present study are labeled as A \& D in Rai (2015) as well.

Figure 4 shows representative grids in the vicinity of the trailing edge in both zones. Both the grids have the same spacing in the wall normal direction at the plate surface. Downstream of the region of interest $(x / D \approx 13.5$ in Cases $A \& D$ and larger $x / D$ values in Cases $E, F \& G)$, the wake grid coarsens gradually in the $x$ direction. This coarsening reduces computational costs and dissipates the wake to a degree that inviscid exit boundary conditions can be employed at the exit boundary of the wake zone.

The wake grid for Case A was constructed with 741 grid points in the streamwise direction, 411 in the cross-stream direction and 256 in the spanwise direction (about $78 \times 10^{6}$ grid points). The resolution achieved along the centerline in the three spatial directions at $x / D=10.0$ is approximately $\Delta x / \eta=3.7$, $\Delta y / \eta=2.2$ and $\Delta z / \eta=2.1$ where $\eta$ is the computed Kolmogorov length scale at the same location. The 
grid resolution in the plate grid in the $\mathrm{x}, \mathrm{y}$ and $\mathrm{z}$ directions for this case is about 17.8, 0.84 and 6.6 wall units respectively, based on the wall shear velocity near the end of the plate. The resolution achieved in all five cases used here is similar. The adequacy of the grid resolution and domain size used is demonstrated in Rai (2013 \& 2014). The computations are all performed at the same freestream Mach number of 0.2. Compressibility effects are quite small at this Mach number.

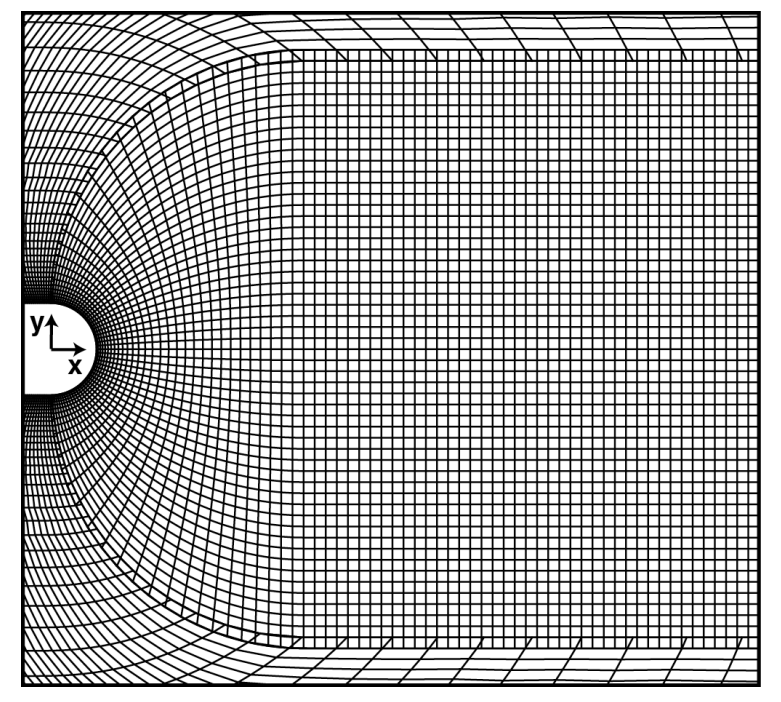

Figure 4. Representative grids in the plate and wake zones in the trailing edge region (Rai 2013).

The primary goal in the current investigation and in Rai (2017) is to study the effect of substantially increasing $\theta / D$ on various characteristics of the wake. Accordingly, the thickness of the plate in Cases $E, F \& G$ was reduced by a factor of $2,8 \& 20$, respectively, from that used in Case $D$. The length of the plate in Cases $E, F$ \& $G$ was the same as in Cases A \& D. In Cases E, F \& G the extent of the computational region in the $z$ direction $(\Delta z)$ in terms of this constant plate length $(L)$ was kept the same as in Case $D$ to ensure that the computed statistics on the plate were not affected by the imposition of periodicity in the $z$ direction. However, the thickness of the plate was reduced as mentioned earlier. This resulted in the spanwise $(\mathrm{z})$ extent of the computational region being $8.0 \mathrm{D}, 32.0 \mathrm{D}$ \& $80.0 \mathrm{D}$ in Cases $E, F \& G$, respectively. The grid resolution in the $z$ direction, however, is the same as in Case $D$. For the same reason (an accurate simulation/representation of the separated boundary layer), the vertical extent of the wake region (in the region where the upper and lower boundaries are horizontal) was also maintained at approximately the same value as in Case D. The same reasoning was also used in the placement of the external boundary of the plate zone and the exit boundary of the wake zone in Cases $E$, $F \& G$ (consequently, in terms of the plate thickness, they were placed approximately $2,8 \& 20$ times further away compared to Case D). Values of $\operatorname{Re}_{\mathrm{D}}$ and the ratio $\psi=(\theta / D) /(\theta / D)_{\text {Case } A}$ for Cases A, D, E, F \& $G$ were provided in Table 1. As mentioned earlier the Reynolds number based on plate length $L$ is the same in all cases, $\operatorname{Re}_{\mathrm{L}}=1.25 \times 10^{6}$.

A high-order accurate upwind-biased method is used here to compute the flow over the plate as well as that in the wake. The convective terms are computed using sixth- and seventh-order upwindbiased finite differences, both with seventh-order dissipation terms. These dissipation terms are the naturally occurring truncation error terms that are obtained in the upwind-biased finite-difference approximation to the convective terms. The viscous terms are computed with fourth-order central differences. The method is iterative-implicit in nature, multiple iterations are employed at each time-step to solve the nonlinear finite-difference equations arising from a fully implicit formulation; the method is second-order accurate in time. The boundaries that contain the computational grids can be classified as natural and zonal boundaries. The natural boundaries include the external boundary of the plate grid, the surface of the plate, the exit boundary of the wake grid, the segments of the upper and lower boundaries 
of the wake grid labeled as "external boundary" in Fig. 3, and, the boundaries in the $z$ direction. The upstream segment of the upper boundary between the plate and wake grids is an example of the zonal boundaries used in the computation. Periodic boundary conditions are imposed on the boundaries in the $z$ direction (homogeneity in $z$ ). No-slip/adiabatic wall conditions are used on the plate surface. Wall blowing/suction is implemented on a short segment on both the upper and lower surfaces of the plate to induce transition to turbulence. The boundary layer is turbulent well upstream of the trailing edge. The upper and lower transitional/turbulent boundary layers and the wake are all computed via DNS. The natural and zonal boundary conditions, and the high-order accurate upwind-biased finite-difference method used here are discussed in detail in the present author's earlier articles cited in Rai (2015).

A test of the adequacy of the resolution used in the wake grid is provided in Rai (2013). A grid resolution study where the grid is refined and convergence of the solution with refinement is demonstrated would be ideal. However, because of the large computational costs involved, the test case utilized is that of a turbulent cylinder wake $\left(\mathrm{Re}_{\mathrm{D}}=3900\right)$. The separating boundaries are laminar but the wake becomes turbulent a few diameters downstream. The computational method is the same as that used here. This case corresponds to the experimental investigation of Ong, Wallace \& Moin (1995). The resolution obtained at $x / D=10.0$ was $\Delta x / \eta=4.1, \Delta y / \eta=3.8$ and $\Delta z / \eta=5.2$ (resolution in the $x$ direction in this cylinder case is approximately the same as in the plate computation, but it is much coarser in the $y$ and $z$ directions for the cylinder). Normal intensity profiles compare well with experimental data at $x / D=10.0$ (Fig. 17 in Rai 2013). The centerline computed velocity spectrum also compares well with experimental data at $x / D=10.0$ over a reduction in magnitude of approximately 500 (Fig. 18 in Rai 2013).

As discussed in Rai (2013) DNS of fully developed turbulent channel flow ( $R e=180$ based on channel half-width) using upwind-biased differences as in the present computation, but with a lower-order of accuracy is provided in Rai \& Moin (1991). The resolution used there in the $x \& z$ directions are about 11.8 \& 5.9 (a direct comparison in the wall normal direction cannot be made because of the staggeredgrid approach that is used in the channel flow DNS, however it is comparable to that used here). The total number of grid points used in the finite difference computation was $3,723,264$. The computed data were compared with data obtained using a spectral method with 3,962,880 grid points (Kim Moin \& Moser, 1987). Intensity profiles across the channel, near-wall behavior of the intensities (intensities normalized by local mean velocity), vorticity fluctuations normalized by mean shear, and skewness factors, obtained in the two simulations, are compared and found to be in excellent agreement. These results show that highorder accurate upwind-biased finite-difference methods yield results that are comparable to those obtained with spectral methods. The upwind-biased differences used for the channel flow were fifth-order accurate (fifth-order dissipation terms). As mentioned earlier, here for the flat plate case we use upwindbiased differences with seventh-order dissipation terms. A comparison of computed boundary layer turbulent intensities with experimental data for Case A (flat plate) is also discussed in Rai (2013). The computed turbulent normal intensities are compared with the experimental data of Karlson \& Johansson (1988) in Rai (2013) and the references cited therein. For example, the average percentage error in rms turbulence intensity ( $u \& v$ components only, at $\operatorname{Re} \theta$ of about 2400 ) is $6.7 \%$. The average percentage error in $w_{r m s}$ at $\operatorname{Re}_{\theta}$ of about 1750 is $4.3 \%$. Here and in the rest of the article $u, v \& w$ are the velocities in the $x, y \& z$ directions, respectively. The computed velocity profile for this case at $90 \%$ plate length compares very well with the log-law and the near-wall linear profile (Fig. 3, Rai 2013). These comparisons clearly demonstrate the adequacy of the grid resolution in the plate grid.

In addition to Case A two more direct numerical simulations were performed in Rai (2014) to (among other reasons) test the adequacy of the computational domain in the $(x, y)$ plane. The Reynolds number based on plate length and diameter are 302,500 and 5000 in both these cases. The second DNS (Grid B, Case B) was performed with boundary placements that are very similar to that of Case A. The external boundary of the plate zone is placed 50.0D from the plate surface (the length of the plate is $60.5 \mathrm{D})$. The exit boundary of the wake zone is $108.0 \mathrm{D}$ from the center of the circular trailing edge and, the vertical extent of this boundary is $81.0 \mathrm{D}$. The spanwise extent of the computational region is $4.0 \mathrm{D}$. The grid resolution is similar to Case A. The third DNS that was performed is similar to that of Case B except that grid points were added as necessary so that the external boundary of the plate zone was placed at 
108.0D instead of 50.0D as in the previous cases. The exit boundary of the wake grid was moved from 108.0D to 207.5D downstream of the trailing edge. The vertical extent of this boundary was increased from $81.0 \mathrm{D}$ to $274.2 \mathrm{D}$. The resolution obtained in the plate and wake grids was very nearly the same as in Case B. The difference in computed values of the fluctuating kinetic energy between the near and far boundary cases, in the near wake, were found to be small, thus demonstrating that boundary placement in the $(x, y)$ plane is adequate. In addition, the phase-averaged, two point-correlation of the randomly fluctuating spanwise vorticity in the $\mathrm{z}$ direction (at vortex cores below the centerline at one particular phase) were obtained for Case A (Fig. 3 in Rai 2014). The correlation is nearly zero by about $\nabla z / D=1.0$ (domain size in the $z$ direction is 4.0D). Thereafter the curves exhibit minor oscillations; this demonstrates the adequacy of the computational domain size used in the $z$ direction. Details regarding resolution and domain size studies can be found in Rai (2013 \& 2014).

\section{FLAT PLATE RESULTS}

The data provided in the following figures were obtained during the data-sampling period (after the initial transients were eliminated). The time-step $n=0$ corresponds to the time at which sampling was initiated. In the following contour plots the colors blue/green represent negative values (deep blue representing the lowest value) of the term/quantity being discussed. Orange, red and magenta represent positive values (magenta bordering on white representing the highest value). Shades of yellow represent values close to zero. The approach taken here is to use the test case that highlights the attribute that is being discussed, followed as necessary by appropriate remarks regarding the same attribute as it appears in other cases.

\subsection{Vortex shedding in Case $F$}

The passage of shed vortices past a given point on the wake center-plane $(y / D=0.0)$ causes fluctuations in the cross-stream velocity $v$. These fluctuations appear as bands of positive and negative contours of $v$ in a (t, z) plane. In Case A (Fig. 7a in Rai 2017) these bands span the entire computational extent in the $z$ direction (this feature is consistent with the shed vortex structure seen in Fig. 1a) and also exhibit considerable small-scale activity because of the turbulent nature of the flow. Figure 5 shows contours of $v$ in a $(t, z)$ plane for Case $F$ over 40 shedding periods $\left(T_{p}\right)$ at $x / D=1.0, y / D=0.0$. A similar plot is provided in Rai (2017) for a smaller extent in $t$ and $z$ (Fig. 17 in this earlier article). A larger extent is provided here to identify the many more high/low intensity shedding events (the time extent of each event of interest is marked by a rectangle In Fig. 5) that were investigated in the present study. The entire spanwise extent of the computational region is included in Fig. 5, and contours corresponding to values of $v$ close to zero have been omitted for clarity.

Here as in Case A we see bands of negative and positive $v$. However, unlike in Case A, but consistent with the vortex structure in Fig. 2, the bands are between 1.0D and 4.0D in spanwise extent. As mentioned earlier the bands suggest intermittency of shedding in time, but that is not the case. The reasons underlying both the recurring high-intensity regions (bands) and the finite extent of the bands in the $z$ direction (attributes that are directly related to the strength/structure of the shed vortices in Fig. 2) are discussed in the following subsection.

The events chosen In Fig. 5 include both high and low intensity shedding periods. To simplify the analysis the set of events only includes those that are well aligned with the $x$ direction (minimal travel in the $z$ direction). In the case of the high-intensity events the rectangles are placed in the central region of the event (in the $z$ direction). Rectangles corresponding to low intensity regions are placed where fluctuation levels are relatively lower than in the surrounding low intensity region. Although all of the events shown in Fig. 5 were investigated in considerable detail including animations of the distribution of pertinent variables, only a few of these events are discussed in detail here (they have been labeled in Fig. 5). Those chosen clearly depict the relevant cause/effect relationship because of the relative simplicity of 
the underlying causative agent. The remaining events are characterized by a multiplicity of these simpler causative agents and are hence more difficult to analyze. The events chosen clearly identify important contributors to the phenomena being investigated.

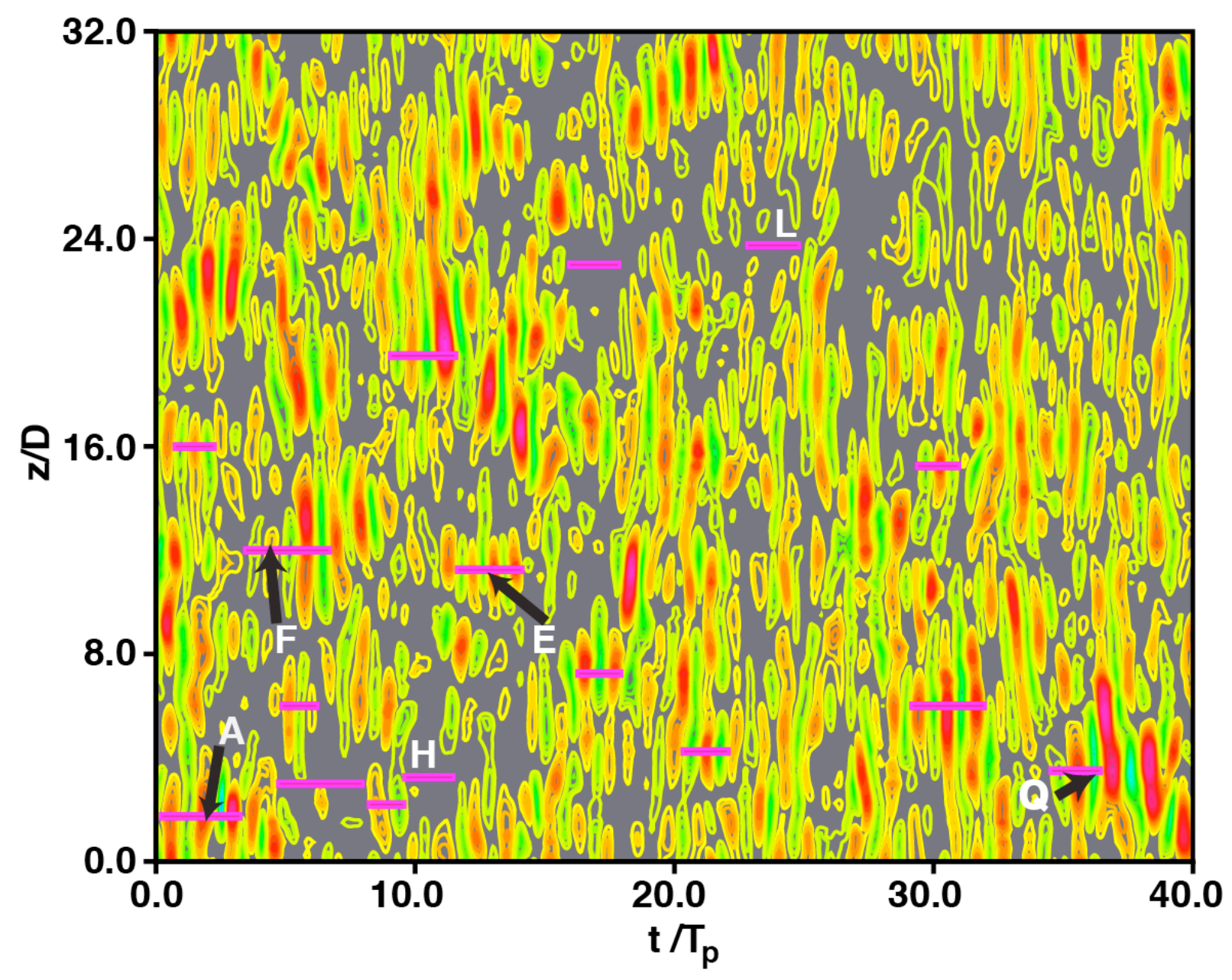

Fig. 5. Contours of cross-stream velocity at $x / D=1.0, y / D=0.0$ over 40 shedding periods.

\subsubsection{Effect of boundary layer vortices on the strength and spanwise extent of shed-vortices}

In Rai (2017) it was hypothesized that boundary layer disturbances may be one of the contributors to the large fluctuations in shed-vortex strength and the related high/low intensity regions in Fig. 5. However, the underlying mechanism was not provided. Here we identify an important contributor (possibly the most important one) to both these fluctuations in intensity and the limited spanwise extent of the high/low intensity regions.

In Rai (2010) the inviscid, incompressible form of the transport equation for spanwise vorticity (Eq. 1 below) was used in investigating the growth of disturbances in the detached shear layers (laminar separation) of a cylinder.

$$
\frac{D \omega_{z}}{D t}=T_{1}+T_{2}+T_{3} \quad ; \quad T_{1}=\omega_{x} \frac{\partial w}{\partial x} ; \quad T_{2}=\omega_{y} \frac{\partial w}{\partial y} ; \quad T_{3}=\omega_{z} \frac{\partial w}{\partial z}
$$


Here $\omega_{x}, \omega_{y}$ and $\omega_{z}$ are the components of vorticity in the $x, y$ and $z$ directions, respectively, and $w$ is the spanwise component of velocity. Assuming that the shear layer is initially quiescent and essentially two-dimensional yields $\omega_{x}=\omega_{y}=0$ and the above equation reduces to:

$$
\frac{D \omega_{z}}{D t}=\omega_{z} \frac{\partial w}{\partial z}
$$

In the cylinder case it was found that the production of $\omega_{z}$ and the subsequent appearance of shear-layer vortices during a shear-layer instability event was a result of a region of positive $\partial w / \partial z$ within the shear layer that is caused by the interaction of the shear layer with recirculation region vortices. This interaction results in an efflux of fluid away from the region of interaction and consequently positive values of $\partial w / \partial z$ locally (and according to Eq. 2, production of $\omega_{z}$ ). The shear-layer vortices are initially oriented with the $z$ direction. They appear on ridges that are a result of the interaction. The development of $\omega_{x}$ that occurs shortly thereafter in the valleys on either side of a ridge results in crescent shaped shear layer vortices; this is also discussed in Rai (2010) utilizing the vorticity transport equation for $\omega_{x}$.

Equation 1 was also used in Rai (2015) to determine the contributors to the growth of detached shear layer instabilities in the case of the thick flat plate with turbulent separating boundary layers. While the reasoning in the case of a cylinder is relatively straightforward, the existence of complex distributions of $\omega_{x}$ and $\omega_{y}$ in a turbulent separating boundary layer makes a similar analysis formidable. Instead the instantaneous distributions of the terms on the right hand side of Eq. 1 were investigated. In Case F, the mean value of $\omega_{z}$ in the very near wake is large while the mean value of the other two components of vorticity is zero. This suggests that shed-vortex strengthening/weakening during certain periods may be occurring because of the contribution of the term $\mathrm{T}_{3}$. It is quite possible that vortices in the separated boundary layer cause a general increase in magnitude in the distribution of $T_{3}$ in the trailing edge region as they convect past this region. Two events were considered to investigate this possibility; one with relatively strong shed vortices and high intensity fluctuations in $v$ (Event $E$ in Fig. 5) and another where the shed vortices are weak and the velocity fluctuations are correspondingly weak (Event $\mathrm{H}$, no bands in Fig. 5). Both these events are 'simple' events; that is, the distribution of $\partial w / \partial z$ and $\omega_{z}$ (constituents of the term $\mathrm{T}_{3}$ ) retain their sign almost always in the region where the shed vortex initially forms and evolves (this was determined via animations). Consequently, the distribution of $T_{3}$ also retains its sign almost always in the early formation region during the period of interest (Events $E$ \& $H$ ).

Figure $6 a$ \& $6 \mathrm{~b}$ show instantaneous in-plane velocity vectors and contours of $\partial w / \partial z$ at two instants in time in a $(x, y)$ plane for Event $E$ ( $z$ location corresponds to center of rectangle in Fig. 5). The upper shed vortex forms and strengthens as it travels downstream during the intervening period. The circulating flow associated with this vortex is evident in these two figures. The shed-vortex related pressure minimum obtained at each of these instants is marked with an X. The center of rotation and the pressure minimum are at slightly different locations at both instants. Subtracting the velocity at the pressure minimum from the velocity field and redrawing the velocity vectors (being in a frame of reference moving with the velocity at the pressure minimum) moves the center of rotation to the location of the pressure minimum and little else.

The contours in Figs. $6 a$ \& $6 \mathrm{~b}$ show that $\partial w / \partial z$ is positive in the region containing the shed vortex at both instants. An animation shows $\partial w / \partial z$ is positive in the shed-vortex formation region during the entire period between these two instants. Consequently the distribution of $T_{3}$ during this period also remains negative in the formation region ( $\omega_{z}$ is negative). Figures $7 \mathrm{a}$ and $7 \mathrm{~b}$ show velocity vectors and the distribution of $T_{3}$ at the same time instants as in Figs. $6 a$ and $6 \mathrm{~b}$, respectively. The negative upper shed vortex is embedded in a region where $T_{3}$ is negative and as a result is strengthened by this term. This of course is entirely consistent with the fact that Event $E$ is a high-intensity event (as seen in Fig. 5). Figures $7 \mathrm{a} \& 7 \mathrm{~b}$ also show that in the region where the subsequent lower positive shed vortex forms (soon after the time instant corresponding to Fig. $7 \mathrm{~b}$ ) the distribution of $T_{3}$ is positive; that is, the 
conditions are appropriate for enhanced vortex shedding. Animations showed that the other shed vortices that form and convect downstream during Event $E$ were also similarly enhanced by the term $T_{3}$.
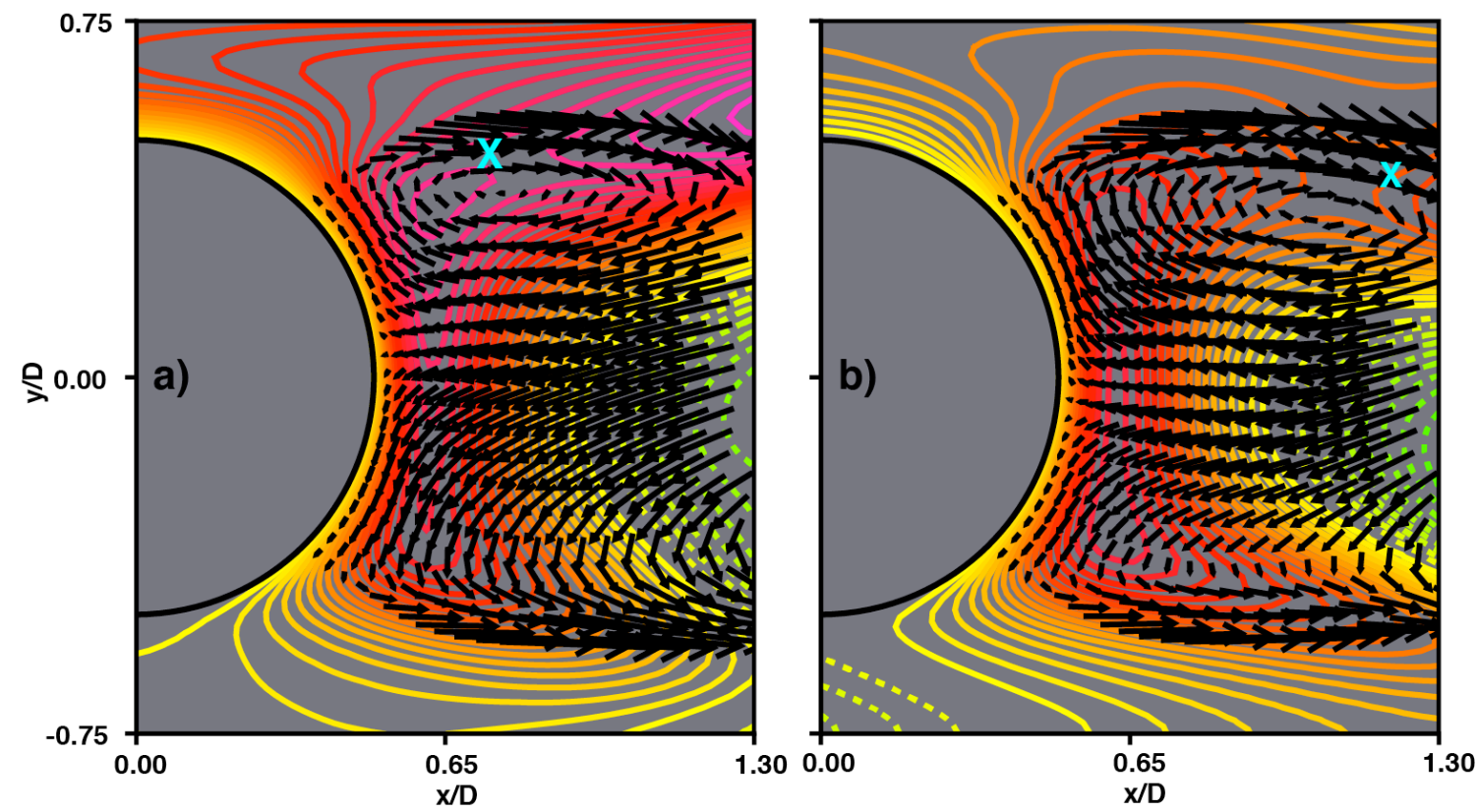

Figure 6. Velocity vectors and contours of instantaneous $\partial w / \partial z$ at a) $n=6040$ and b) $n=6140$, Event E.
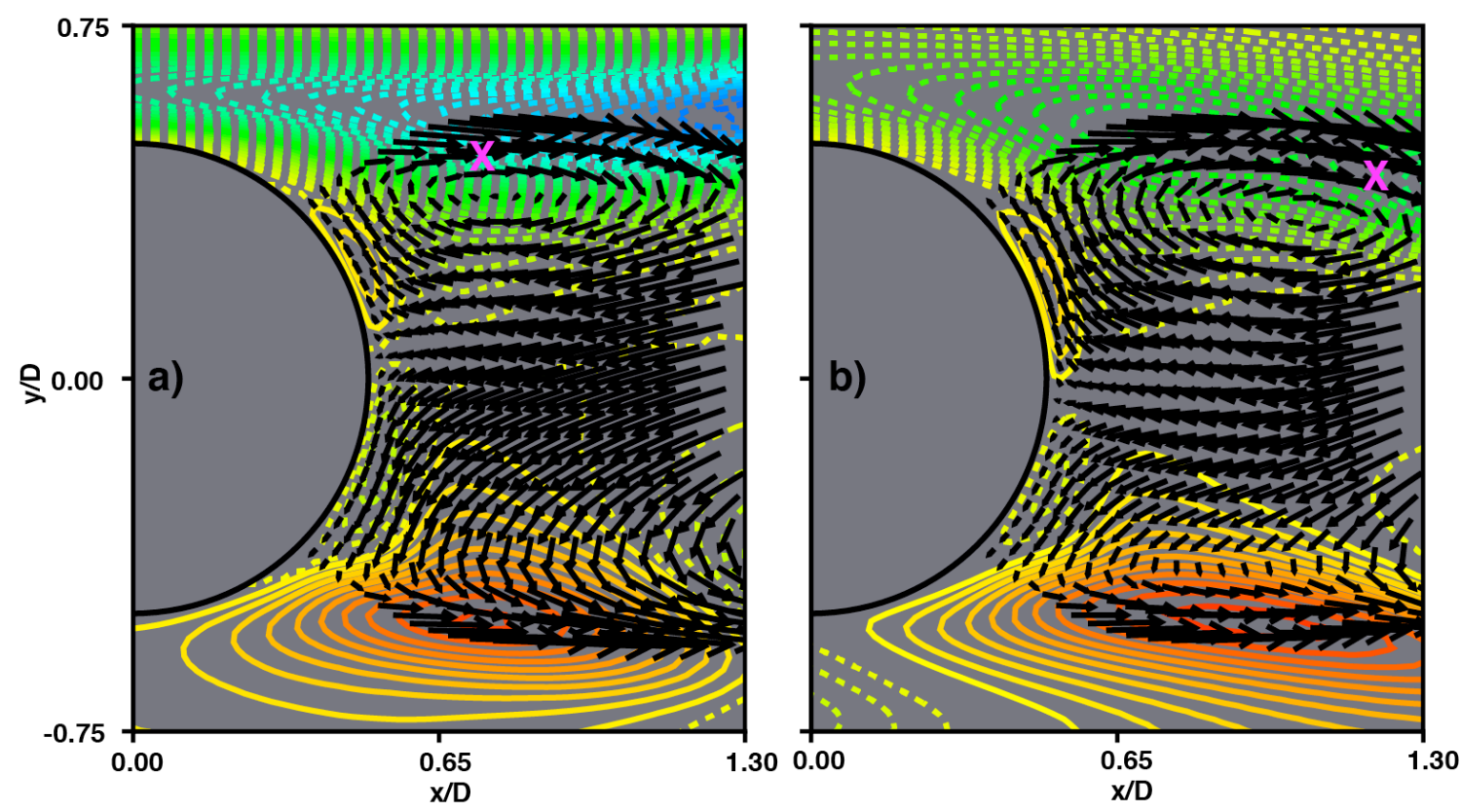

Figure 7. Velocity vectors and contours of instantaneous $T_{3}$ at a) $n=6040$ and b) $n=6140$, Event $E$.

The origin of the region of positive $\partial w / \partial z$ in the formation region of the upper shed vortex (and subsequently the lower shed vortex) is now investigated. Figures $8 \mathrm{a}-8 \mathrm{~d}$ show in-plane velocity vectors and contours of the variables of interest in an end-plane located at $x / D=1.0$ in the region $-2.0 \leq y / D \leq 2.0$, $9.0 \leq z / D \leq 14.0$ (looking upstream from a location $x / D>1.0$ ). The time instant chosen corresponds to a time between the two instants of Figs. $7 \mathrm{a} \& 7 \mathrm{~b}$, when the center of the upper shed vortex is located at approximately $x / D=1.0$. The vertical white line shows the $z$ location of the center of Event $E$ (see Fig. 5). 


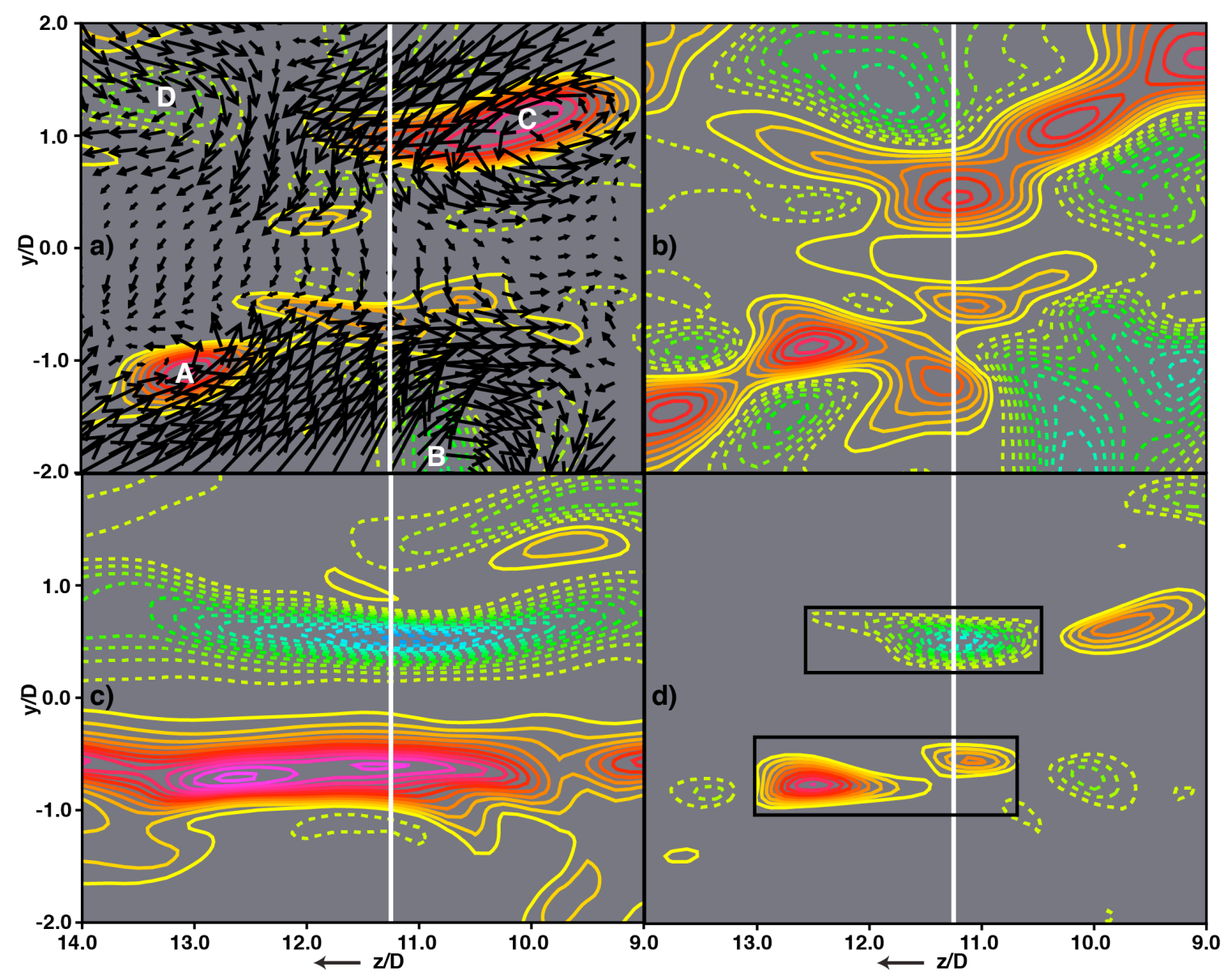

Figure 8. Contours (instantaneous) in an end-plane at $x / D=1.0$ at $n=6080 ; a) \omega_{x}$ with in-plane velocity vectors $(w, v)$ at $12.5 \%$ of sampling points, b) $\partial w / \partial z c) \omega_{z}$ and d) $T_{3}$; vertical white line is at the $z$ location corresponding to the center of Region $\mathrm{E}$.

Figure 8a shows instantaneous in-plane velocity vectors $(w, v)$ and contours of instantaneous streamwise vorticity, $\omega_{x}$. Four of the dominant (largely) streamwise vortices $(A, B, C, D)$ in the separated boundary layer (they all occur mostly in the region lyl >1.0) are marked in Fig. 8a. Together they result in the in-plane induced velocity distribution seen via the velocity vectors. The resulting distribution of $\partial w / \partial z$ is provided in Fig. $8 \mathrm{~b}$. In the vicinity of the white line, in the region $-1.0 \leq y / D \leq 1.0, \partial w / \partial z$ is positive. Figure $8 \mathrm{c}$ shows the distribution of $\omega_{z}$ at the same instant. The upper and lower detached shear layers are seen as bands of contours (opposite sign) running the length of the figure ( $z$ direction). The distribution of the term $T_{3}$ resulting from the distributions of $\partial w / \partial z$ and $\omega_{z}$ are provided in Fig. 8d. The islands of shed-vortex enhancing distributions of $\mathrm{T}_{3}$ (negative above and positive below) are observed within the rectangles. Similar distributions of $T_{3}$ in the vortex formation region were observed during the entire period of Event $E$. Thus this event (E) lends credence to the hypothesis that vortices in the separated boundary layer can strengthen shed vortices. In addition, the finite extent of the vortexenhancing regions of $T_{3}$ in the z-direction (roughly $2.0 \mathrm{D}$ in Fig. $8 \mathrm{~d}$ ) can be expected to result in bands associated with Event $E$ that have the same extent in the z-direction (also roughly 2.0D as in Fig. 5).

To visualize the structure of the vortices in the boundary layer (prior to and soon after separation), contributing to the production of positive $\partial w / \partial z$ in the region containing Event $E$, surfaces of constant $\lambda_{2}$ (negative) colored by the sign of the streamwise vorticity were obtained and are provided in Fig. 9 . They are at the same instant as in Fig. 8 and show the vortices below the lower surface of the plate (vortices 
marked $A$ and $B$ in Fig. $8 \mathrm{a}$ ). In the region of the end-plane at $x / D=1.0$ the boundary layer vortices are largely streamwise. They result in the induced velocity field seen in Fig. 8a, the corresponding distribution of $\partial w / \partial z$ in Fig. $8 \mathrm{~b}$, the consequent distribution of $\mathrm{T}_{3}$ in Fig. $8 \mathrm{~d}$ and ultimately enhanced vortex shedding during Event $\mathrm{E}$. The distance between the centers of the streamwise vortices in the vicinity of the endplane is about 3.0D. The vortex enhancing velocity field (positive $\partial w / \partial z$ ) lies approximately between these two centers. This is one of the important contributors to the finite extent of the bands seen in Fig. 5. It is also important to note that if the z-locations of the positive streamwise vortex $(A)$ and the negative streamwise vortex $(B)$ in Figs. $8 a \& 9$ were reversed the resulting $\partial w / \partial z$ field would be negative in the region of interest and vortex shedding would not be enhanced but perhaps subdued instead. This point very naturally introduces the next case, that is, Event $\mathrm{H}$ where shedding is greatly subdued.

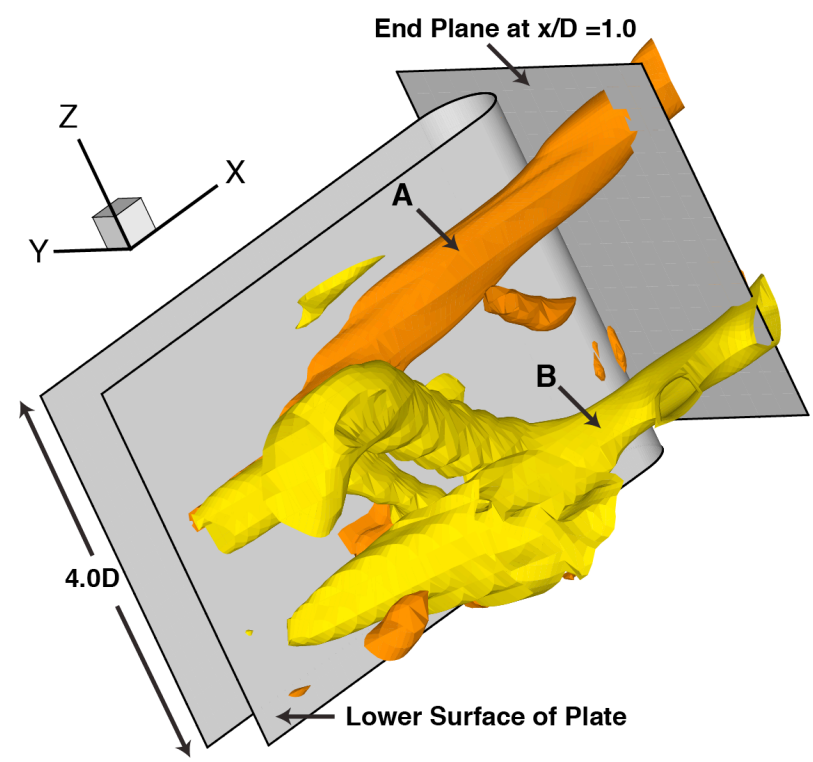

Figure 9. Perspective view of surfaces of constant $\lambda_{2}$ (negative) below the lower plate surface, colored by the sign of streamwise vorticity ( $A$ is positive and $B$ is negative), at $n=6080$, Event $E$.

Figures $10 \mathrm{a} \& 10 \mathrm{~b}$ show instantaneous in-plane velocity vectors and contours of $\partial w / \partial z$ in a $(x, y)$ plane at two instants in time corresponding to Event $\mathrm{H}$ in Fig 5. The z-location of this plane corresponds to the center of the rectangle associated with Event $\mathrm{H}$. Of interest is the formation and evolution of the lower shed vortex. The location of the shed-vortex related pressure minimum is once again marked with an $\mathrm{X}$ in Fig 10b; there was no pressure minimum at the earlier instant (Fig. 10a). The region of circulating flow in Fig. 10a is highlighted with a rectangle. Here, unlike in Event $E$ (Figs. 6a \& 6b), $\partial w / \partial z$ is negative in the vortex formation region. The corresponding distributions of $T_{3}$ at the two time instants are provided in Figs. 11a \& 11b. Clearly $T_{3}$ is negative in the region of formation of the lower positive shed vortex and thus weakens this vortex. Additionally Fig. 11b indicates that the subsequent upper negative shed vortex will also be weakened (region of positive $T_{3}$ above the wake centerline); as in Event $E$, animations show the weakening effect of $T_{3}$ during the event $(H)$. This results in weak velocity fluctuations $(v)$ at the wake centerline (no vertical bands corresponding to event $\mathrm{H}$ in Fig. 5).

As in Event $E$, we investigate the vortices of the separated boundary layer that may be causing the vortex weakening $\partial w / \partial z$ field. Similar to Figs. $8 \mathrm{a}-8 \mathrm{~d}$, Figs. $12 \mathrm{a}-12 \mathrm{~d}$ show velocity vectors and contours of the variables of interest in an end-plane located at $x / D=1.0$ in the region $-2.0 \leq y / D \leq 2.0,0.0$ $\leq z / D \leq 5.0$. The time instant chosen corresponds to a time between the two instants of Figs. 11a \& 11b, when the center of the lower shed vortex is located at approximately $x / D=1.0$. The vertical white line shows the $z$ location of the center of Event $H$ (see Fig. 5). Figure 12a shows in-plane velocity vectors (w, v) and contours of instantaneous streamwise vorticity, $\omega_{x}$. Two of the dominant (largely) streamwise 
vortices (A, B) below the wake centerline and in the separated boundary layer (they mostly occur in the region $y / D<-1.0)$ are marked in Fig. 12a. Together they result in the in-plane induced velocity distribution seen via the velocity vectors below the centerline $(y / D=0.0)$. The resulting $\partial w / \partial z$ distribution, shown in Fig. $12 \mathrm{~b}$, is negative in the vicinity of the white line below the centerline (and in addition, above centerline). Figure 12c, as in Fig. 8c, shows the distribution of $\omega_{z}$. The resulting distribution of the term $T_{3}$ is provided in Fig. 12d. Clearly this term has a vortex weakening effect both above and below the centerline in the corresponding vortex formation regions (see rectangles). This again is in accordance with the original hypothesis that boundary layer vortices may strengthen or weaken shed vortices. The spanwise extent of the shed-vortex weakening regions in Fig. 12d is also finite as in Event E.
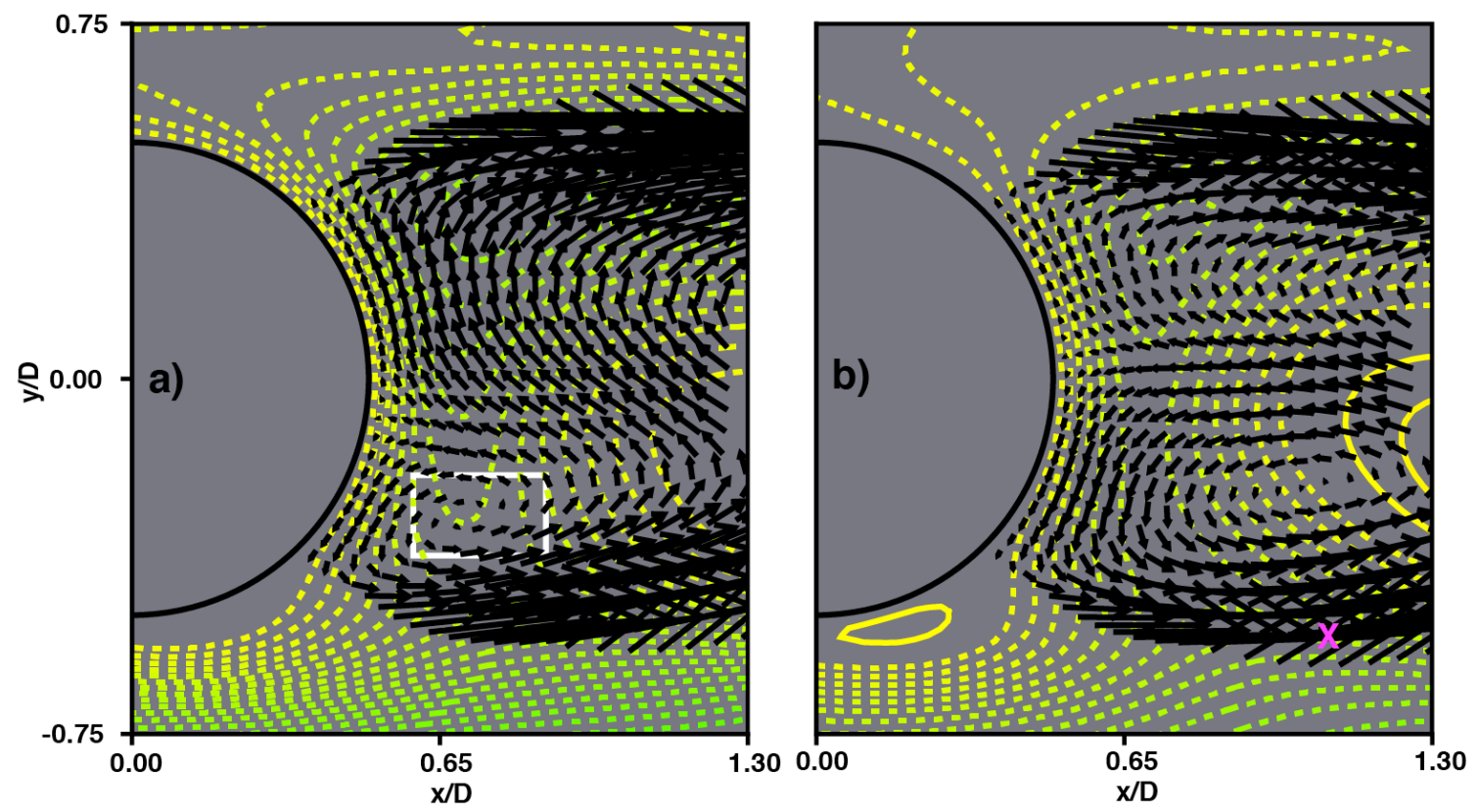

Figure 10. Velocity vectors and contours of instantaneous $\partial w / \partial z$ at a) $n=4780$ and b) $n=4920$, Event $H$.
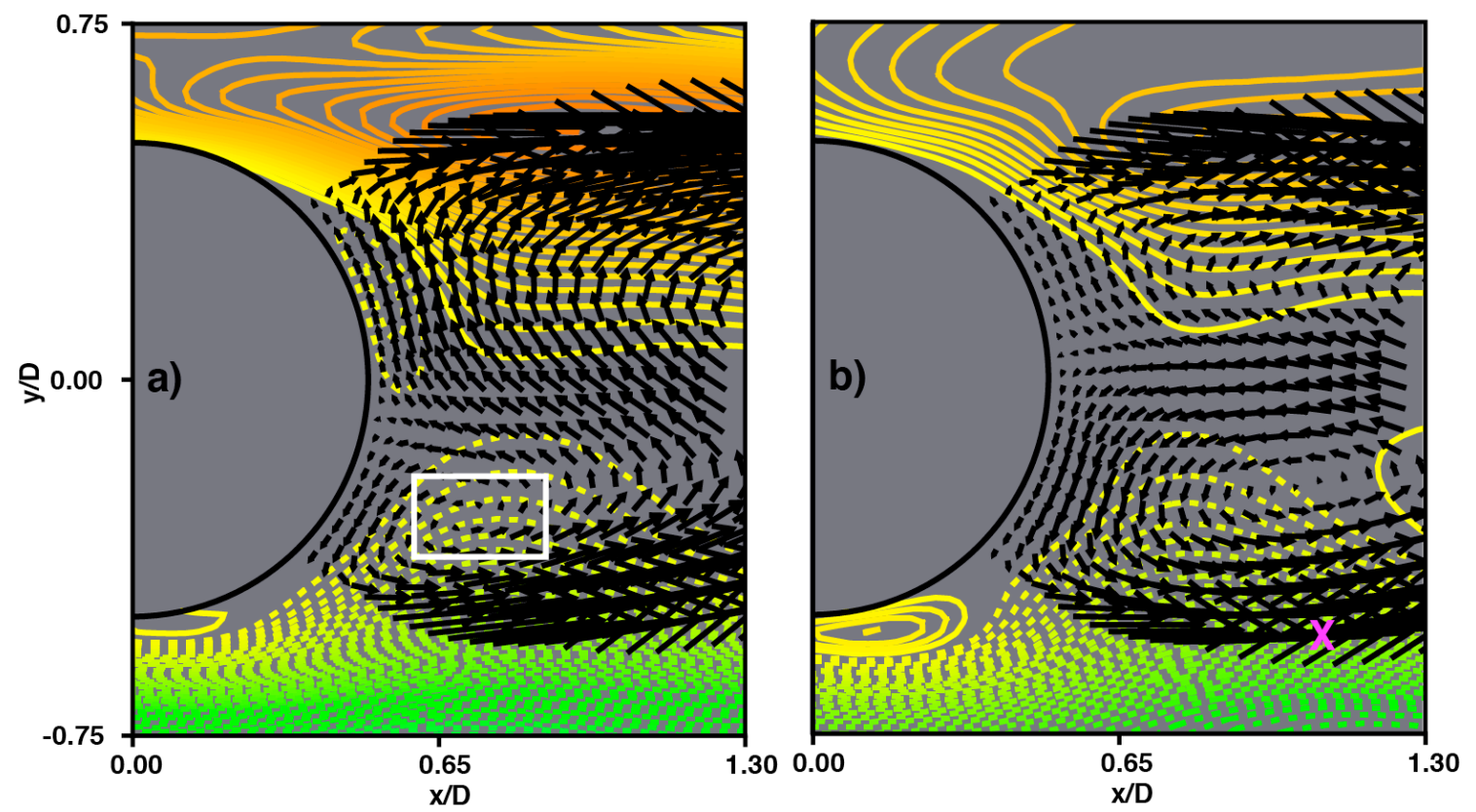

Figure 11. Velocity vectors and contours of instantaneous $T_{3}$ at a) $n=4780$ and b) $n=4920$, Event $H$. 


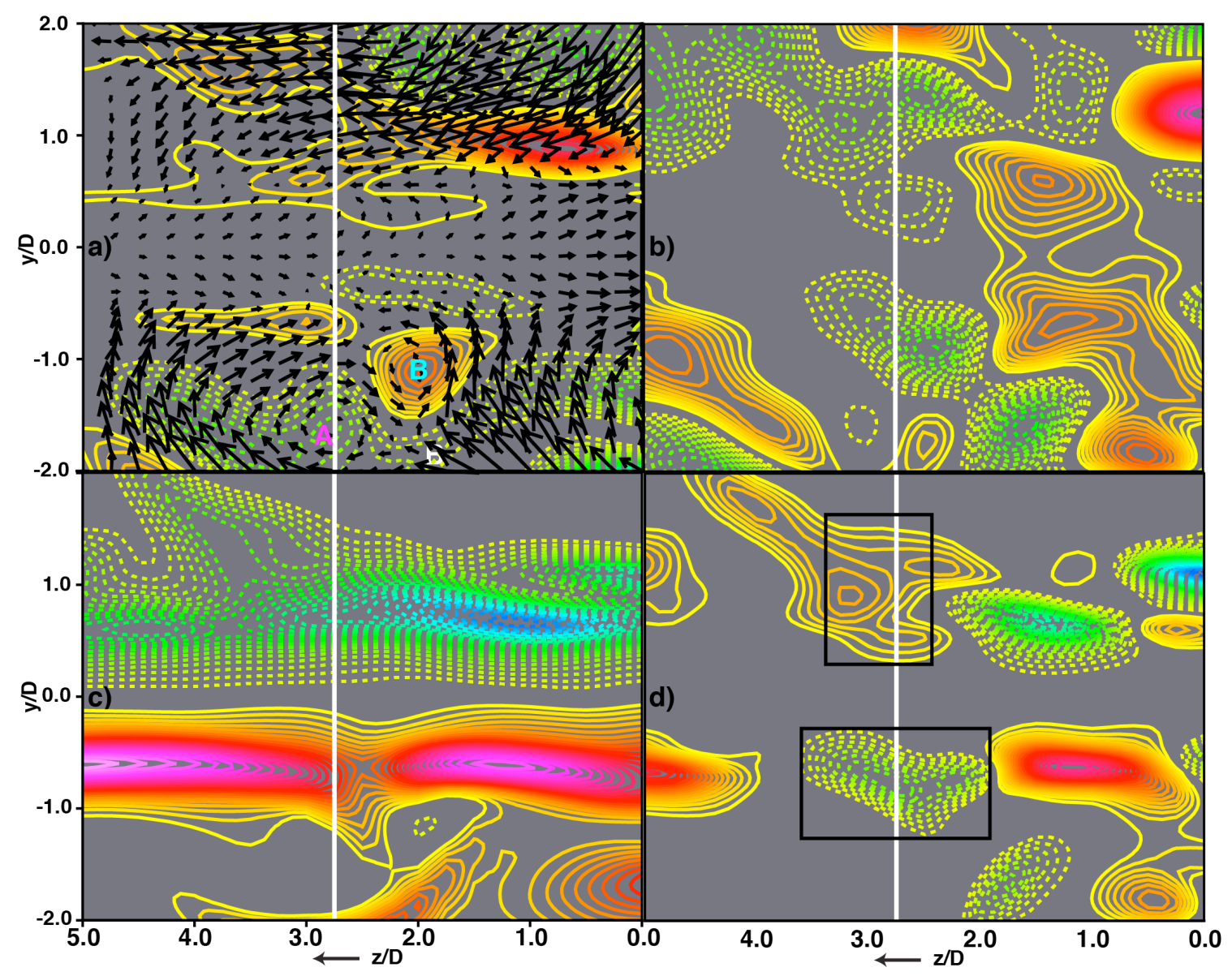

Figure 12. Contours (instantaneous) in an end-plane at $x / D=1.0$ at $n=4840$; a) $\omega_{x}$ with in-plane velocity vectors $(w, v)$ at $12.5 \%$ of sampling points, $b) \partial w / \partial z c) \omega_{z}$ and d) $T_{3}$; vertical white line is at the $z$ location corresponding to the center of Region $\mathrm{H}$.

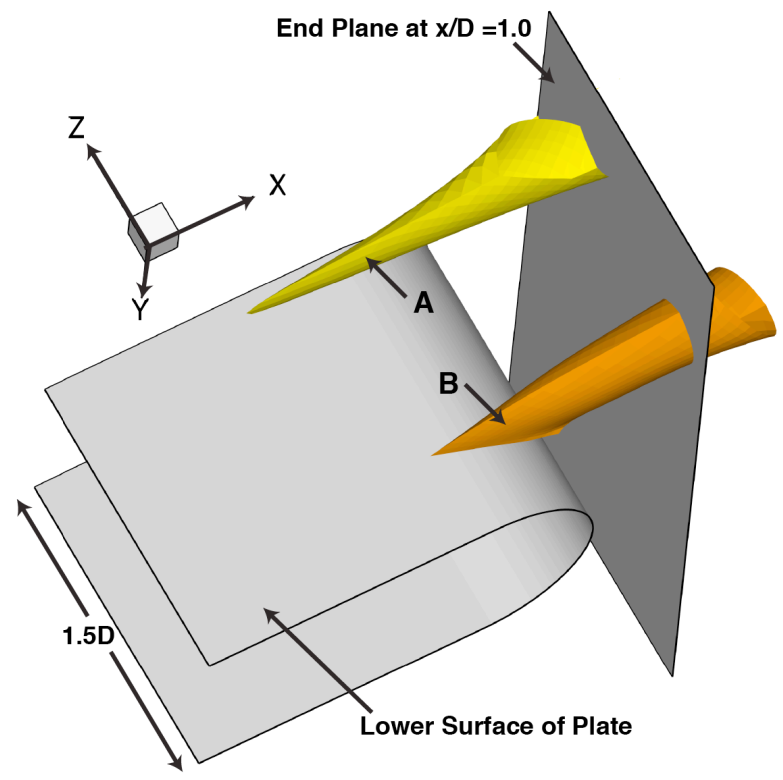

Figure 13. Perspective view of surfaces of constant $\lambda_{2}$ (negative) below the lower plate surface, colored by the sign of streamwise vorticity, at $\mathrm{n}=4840$, Region $\mathrm{H}$. 
Figure 13 shows surfaces of constant $\lambda_{2}$ (negative) colored by the sign of the streamwise vorticity. They are at the same instant as in Fig. 12 and show the vortices below the lower surface of the plate (vortices marked A and B in Fig. 12a). Here, as in Event E, we have a pair of streamwise vortices that result in an induced velocity field that has a significant effect on the strength of the shed vortices during an event. Unlike in Fig. 9 the positive streamwise vortex occurs at a lower z-location; the resulting $\partial w / \partial z$ field in the region of interest is negative and we have greatly weakened shed vortices.

The foregoing discussion focused on the shedding of a single vortex during an event of interest. The information regarding the other shed vortices that together constitute the entire event was obtained via animations and reported. Now we explore the possibility of time-averaging the distribution of $T_{3}$ over an entire event to determine if this time-average corresponds to the observed behavior during the event (enhanced or subdued shedding). Figure 14 shows the event-time-averaged distributions of $T_{3}$ obtained for four events; two with enhanced shedding ( $E \& Q$ ) and two with subdued shedding $(H \& L)$. The factor $F$ shown in each of the subplots represents the number that the values of $T_{3}$ were multiplied by so that the features of interest are easily observed (specified $\mathrm{min} / \mathrm{max}$ values and the number of contours plotted after this modification are the same for each subplot). The distributions obtained for Events $E$ and Q (Figs. $14 a \& 14 b)$ show negative values of $T_{3}$ in the vortex formation region above the centerline and positive values below the centerline. The opposite is true for Events $H$ \& $L$ (Figs. 14c \& 14d). This is completely consistent with the observed behavior, that is, enhanced shedding in Events $E$ \& $Q$ and subdued shedding in Events $\mathrm{H}$ \& L.

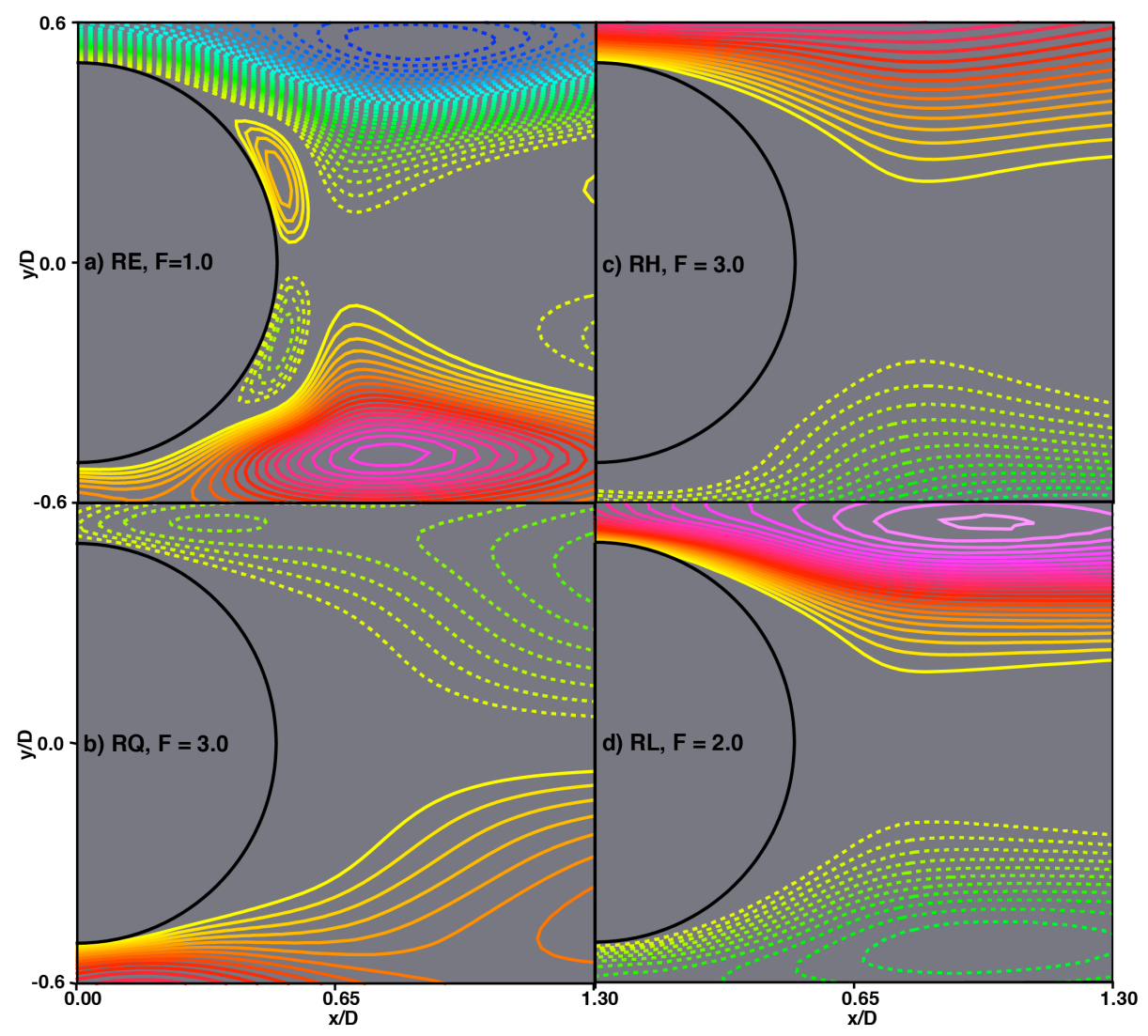

Figure 14. Contours of event-time-averaged $\mathrm{T}_{3}$ for a) Event $\mathrm{E}$, b) Event $\mathrm{Q}, \mathrm{c}$ ) Event $\mathrm{H}$ and d) Event L.

Although the distributions in Figs. $14 \mathrm{a}-14 \mathrm{~d}$ are consistent with the overall behavior within an event, the enhancing/weakening nature of the event-time-averaged $T_{3}$ term does not guarantee the 
strengthening or weakening of every shed vortex that forms and evolves during an event. Strengthening may, for example, occur over a portion of a particular shed vortex for a fraction of the period of time it occupies the trailing edge region. This aspect is of course not reflected in a distribution of the event-timeaverage of the term $\mathrm{T}_{3}$. Despite this limitation plots of the kind shown in Fig. 14 are of utility in identifying events of interest; this of course needs to be followed by an in-depth event analysis via animations etc. Other complexities such as weakening of the upper shed vortices and strengthening of the lower shed vortices during an event, or vice versa, may also occur. It was found both via animations and, plots of time-averaged quantities that the contribution of the term $T_{1}$ was small compared to that of $T_{3}$ and, while $T_{2}$ was in a few instances comparable to $T_{3}$, overall, the term $T_{3}$ played a dominant role. This is not surprising since the mean value of $\omega_{x}$ and $\omega_{y}$ is zero and that of $\omega_{z}$ is large in magnitude in the vortex formation region.

It is abundantly clear that the wake flow in Case $F$ is exceedingly complex. Nevertheless, much can be gleaned from the "simple" events and applied to obtain a better understanding of the more complex events (where there are a multiplicity of simple causative agents). Here we have demonstrated that streamwise vortices in the separated boundary layer that are in close proximity to the shed vortices have a significant effect on the strength/structure of these shed vortices when $\theta / D$ is large.

\subsubsection{Contributors to the variation in shedding period}

As observed in Rai (2017) the contours of cross-stream velocity in the wake center-plane (fig. 5 in the present article) show that the shedding period (width of pairs of vertical bands) varies from event to event (sometimes even within an event). In some instances the ratio of shedding periods between events is significant; ratios as high as 1.6 (between events), have been observed. A probable cause is the turbulent nature of the separating boundary layer. Fluctuations in the streamwise velocity within the boundary layer could slow down or hasten the convective rate of shed vortices that are confined to the detached shear layer (as in Case F) and thus increase/decrease the shedding period. A first analysis of this phenomenon was conducted in Rai (2017) and the potential of boundary layer high/low-speed streaks to significantly alter shedding frequency during an event was noted. A more detailed investigation of this phenomenon is provided in the present investigation. Extensive use is made of the distribution of the fluctuating component of the streamwise velocity $\left(u^{\prime}\right)$ within the boundary layer (at and upstream of $x / D=$ 0.0 , and close to the plate surface at $l y-y_{\text {wall }} l / D=0.25$ ) and, also in the near wake, in this analysis.

Figures $15 \mathrm{a} \& 15 \mathrm{~b}$ show contours of instantaneous $\mathrm{u}^{\prime}$ just above the upper surface of the plate $(\mathrm{x}$, $z$ plane), at the beginning and end of Event E (Fig. 5), respectively. The white rectangle in these two subplots is located at the $z$ location corresponding to Event $E$ (see Fig. 5). In Fig. 15a there is a highspeed streak approaching the trailing edge from left to right. This high-speed streak travels over the trailing edge region and the vortex formation region in time. Figure 15b shows the tail end of the same streak at the end of Event E. Figures $15 c \& 15 d$ show contours of instantaneous u' just below the lower surface of the plate, at the beginning and end of Event E, respectively. Here again we have a high-speed streak travelling over the trailing edge and the vortex formation region. Clearly both the upper and lower boundary layers both have the potential to accelerate the shedding process. In contrast, in Event $F$, figures similar to Figs. $15 \mathrm{a}-15 \mathrm{~d}$ show low-speed streaks in both the upper and lower boundary layers in the region corresponding to this event. Thus there is the potential in Event $F$, for a slowing of the shedding process. As a matter of fact the ratio of shedding periods between Cases $F \& E$ is 1.55 .

A time-shift corresponding to the approximate time required for the flow features in the boundary layer to convect from $X / D=0.0$ to $x / D=1.0$ (where the shed vortices first appear) has been accounted for in obtaining Figs. $15 a-15 d$. This time-shift was obtained by tracking easily observed features in animations of $u^{\prime}$ in a ( $\left.x, z\right)$ plane above the plate surface (as in Figs 15a \& 15b). 


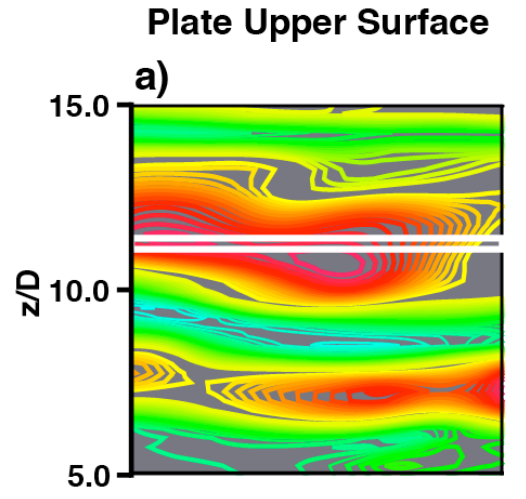

b)

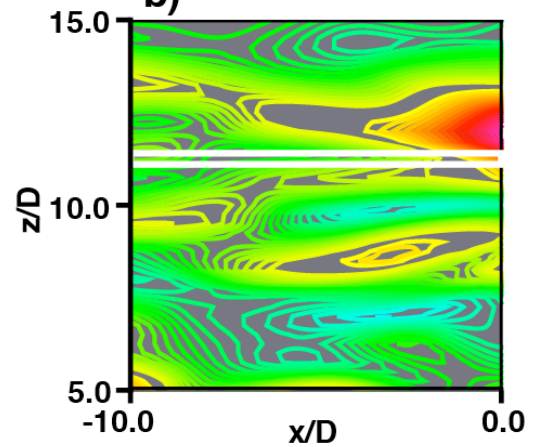

Plate Lower Surface

c)

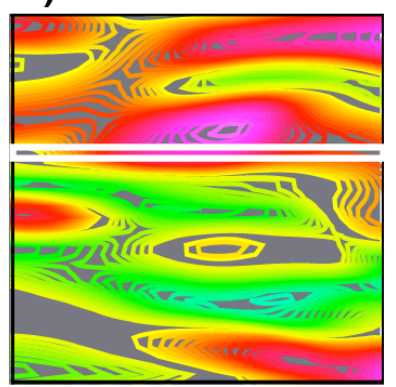

d)

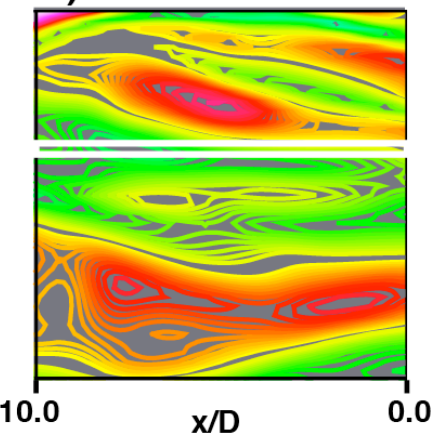

Figure 15. Contours of instantaneous $u^{\prime}$ at $l y-y_{\text {wall }} / \mathrm{D}=0.25$, a) \& c) upper \& lower plate surfaces, respectively at the beginning of Event $E, b) \&$ d) upper \& lower plate surfaces at the end of Event $E$.

To more clearly illustrate the effect of high speed streaks on shedding period Fig. 16a shows the time variation of $u^{\prime}$ at $x / D=1.0$ in the region $-0.75 \leq y / D \leq 0.75$ (vortex formation region) for Event $E$. The $z$-location of this subplot corresponds to the z-center of Event $E$ (see rectangles in Figs. 5 \& 15).

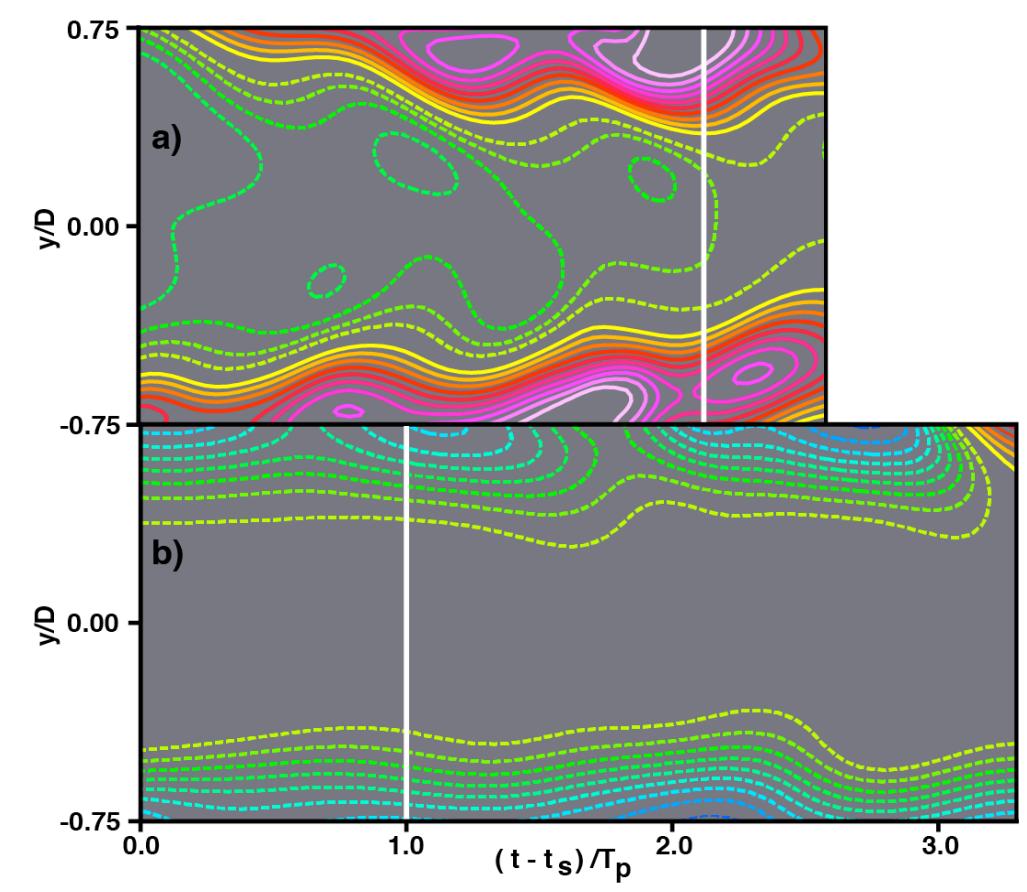

Figure 16. Contours of $u^{\prime}$ in $a(t, y)$ plane at $x / D=1.0$ in a) Region $E$ and b) Region $F$. 
The left boundary of Fig. 16a, (and also the time instant $t_{s}$ ), correspond to the beginning of Event $E$. The regions containing red contours (positive $\mathrm{u}^{\prime}$ ) in Fig. $16 \mathrm{a}$ in the vortex formation region (roughly lyl/D > $0.45)$ are clearly observed for Event E. Figure 17a shows instantaneous in-plane velocity vectors and contours of instantaneous $u^{\prime}$ for Event $E$ in a $(x, y)$ plane (same z-location as in Fig. 16a). Figure 17a was obtained at the time instant corresponding to the vertical line in Fig. 16a. The upper shed vortex (associated pressure minimum marked with an $\mathrm{X}$ ) is largely embedded in a region of positive $\mathrm{u}^{\prime}$ and thus travels downstream more rapidly causing the shedding period to decrease. Figure $17 \mathrm{a}$ also shows the formation of the subsequent lower vortex (seen within the rectangle in Fig. 17a and more clearly in Fig. 17b). This newly shed vortex is also subjected to a relatively rapid convection rate as it evolves. An animation and Fig. 16a show that Fig. 17a is characteristic of the entire Event $E$. In this sense, Event $E$ is a 'simple' event.

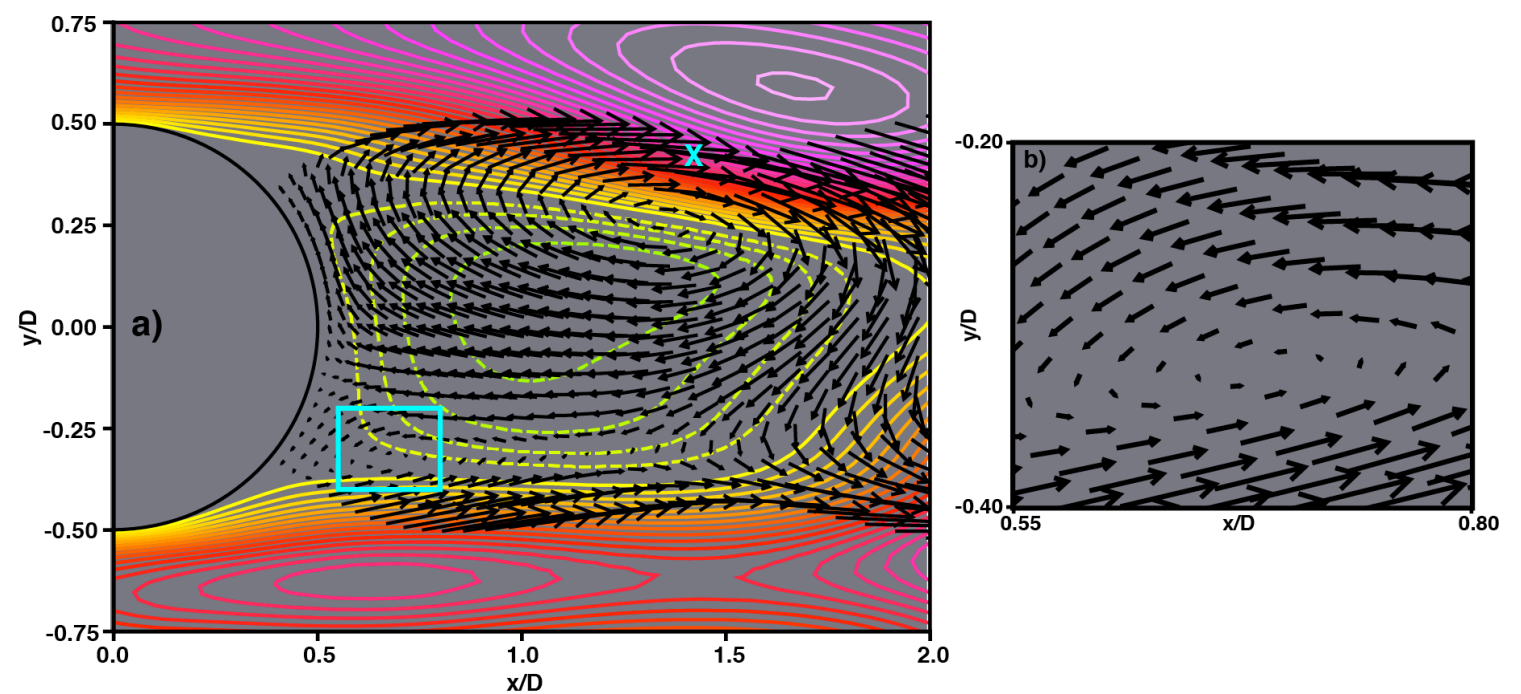

Figure 17. a) Instantaneous velocity vectors and contours of $\left.u^{\prime}, b\right)$ enlarged view of the region of formation of lower shed vortex showing instantaneous velocity vectors, Event $E$.

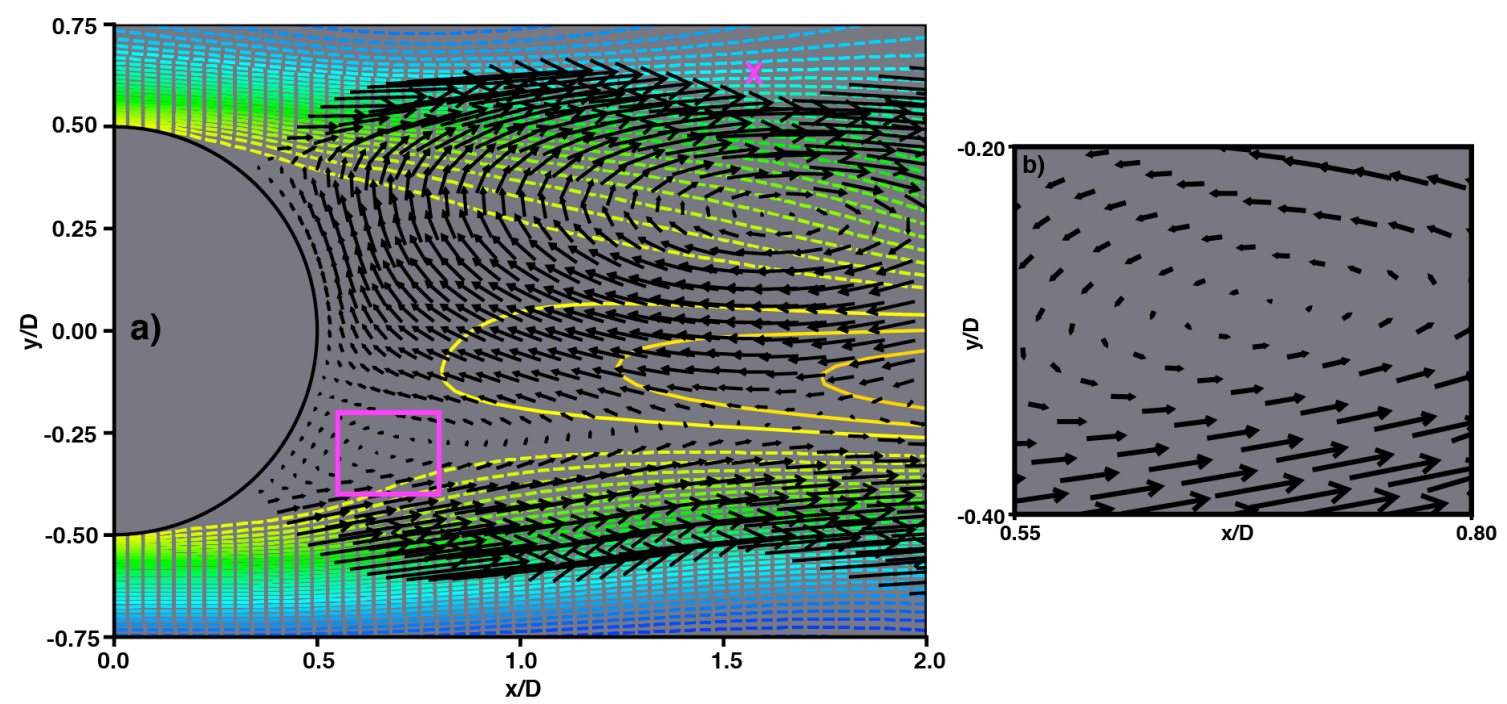

Figure 18. a) Instantaneous velocity vectors and contours of $\left.u^{\prime}, b\right)$ enlarged view of the region of formation of lower shed vortex showing instantaneous velocity vectors, Event $F$.

Figure $16 \mathrm{~b}$ is the counterpart of Fig $16 \mathrm{a}$ for Event $\mathrm{F}$. Here in the vortex formation region we have negative values of $u^{\prime}$. Figure $18 a$ is the counterpart of Figure $17 a$, but for Event $F$. The upper shed vortex 
in Fig. 18a, with its associated pressure minimum (marked with an X), is embedded in a region of negative $\mathrm{u}^{\prime}$ thus resulting in a slower than average vortex convection rate. Figure 18a also shows the formation of the subsequent lower shed vortex (see rectangle in Fig. 18a and also Fig. 18b). In time, this vortex evolves and travels downstream at a lower than average speed. An animation and Fig. 16b show that Fig. 18a is characteristic of the entire Event $F$; this is consistent with the larger than average shedding period that this event exhibits. Event $F$ is also a 'simple' event in the sense of Event $E$.

Figure 19 shows the event-time-average of $u^{\prime}$ as a function of $y / D$ at $x / D=1.0$ for Events $E$ and $F$ (time-average of the time-variation seen in Fig. 16). The difference between the two curves is remarkable. The one obtained for Event E clearly shows the potential for accelerated shedding at both ends (roughly lyl/D $>0.45$ where the vortices form and evolve) and its counterpart for Event $F$ shows the potential for decelerated shedding at the two ends.

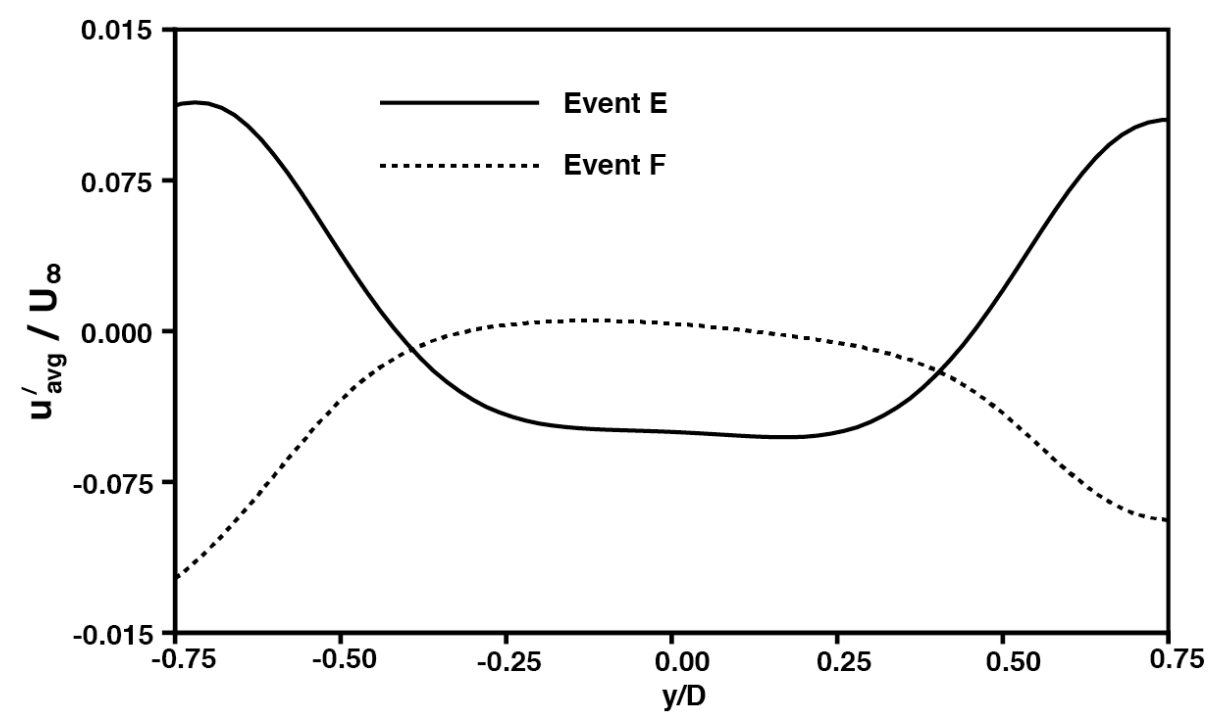

Figure 19. The event-time-average of $u^{\prime}$ as a function of $y / D$ for Events $E$ and $F$.

Events $E$ and $F$ are simple in the sense that both upper and lower boundary layers tend to either hasten the shedding process (Event E) or slow it down (Event F) over the entire event. However, many of the events depicted in Fig. 5 exhibit more complicated behavior. Figure 20 shows the time variation of $\mathrm{u}^{\prime}$ at $x / D=1.0$ in the region $-0.75 \leq y / D \leq 0.75$ (vortex formation region) for Event A. Unlike Figs. 16a \& 16b, the region above the wake centerline $(y / D>0.0)$ shows both positive and negative regions of $u^{\prime}$. The region below the centerline is 'simple'; it increases the shedding period of the lower shed vortices.

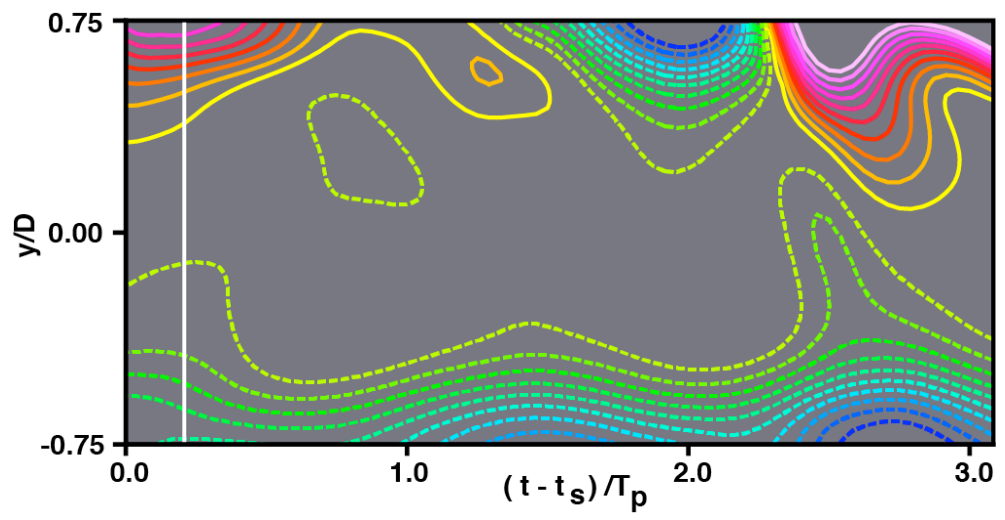

Figure 20. Contours of $u^{\prime}$ in a $(t, y)$ plane at $x / D=1.0$ for Event $A$. 
Figure 21 shows instantaneous velocity vectors and contours of $u^{\prime}$ for Event $A$ at the time instant corresponding to the vertical white line in Fig. 20. As can be expected from the upper positive region in Fig 20 (at this instant), the upper shed vortex in Fig. 21a, with its associated pressure minimum (marked with an $\mathrm{X}$ ) is largely embedded in a region of positive $\mathrm{u}^{\prime}$. Thus the upper shed vortex travels downstream relatively rapidly. However, the subsequent lower shed vortex takes more time than average to evolve (see rectangle in Fig. 21a and also Fig. 21b) because it becomes embedded in a region of negative $u^{\prime}$ as it travels downstream. Figure 21a shows the sharply contrasting effects of the upper and lower detached shear layers at the chosen instant in time (above and below the wake centerline). The 'simple' behavior seen in Figs. $17 \mathrm{a}$ and $18 \mathrm{a}$ for Events $\mathrm{E}$ and $\mathrm{F}$, respectively, is absent here. Simultaneous acceleration and deceleration of shedding is present. In this particular case the event shedding period is larger than average because of the preponderance of negative $u^{\prime}$ in the region (as seen in Fig. 20).

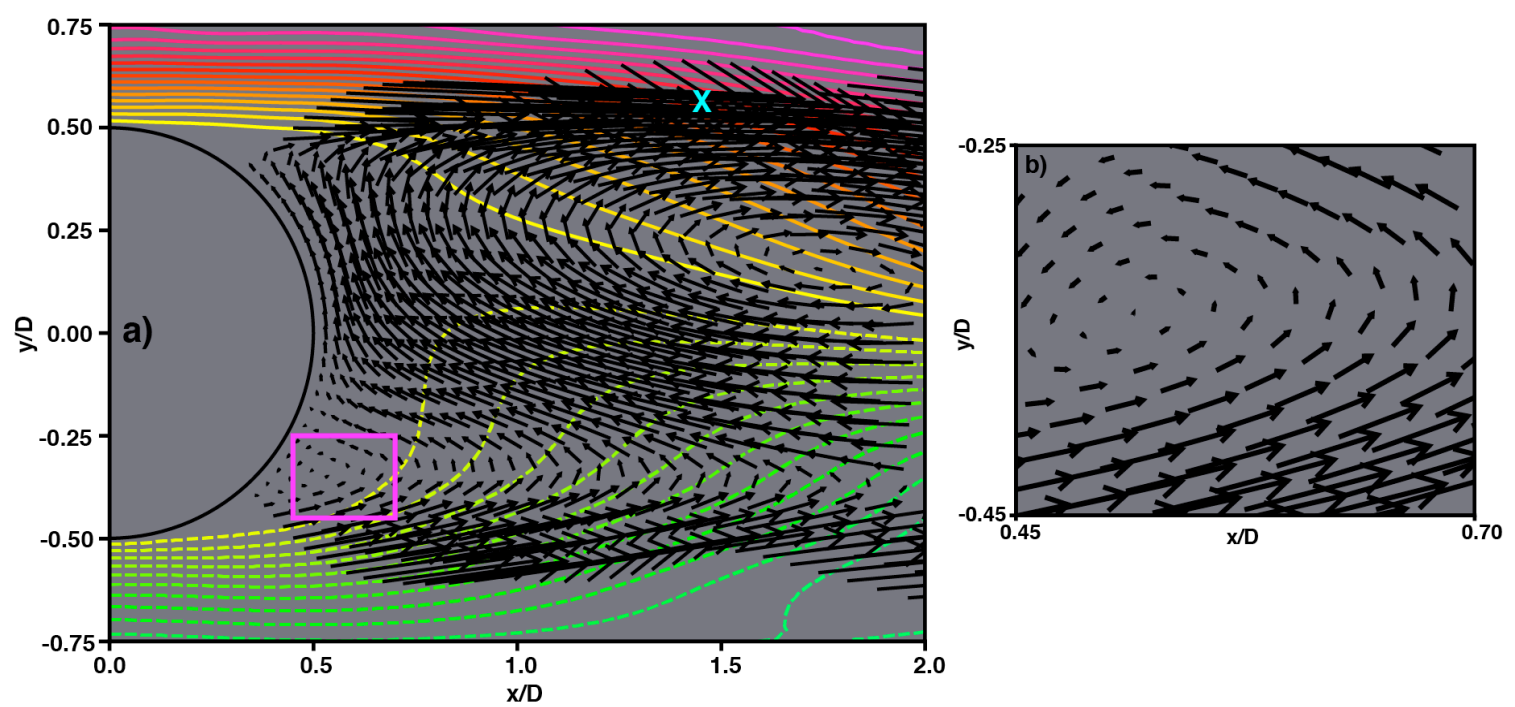

Figure 21. a) Instantaneous velocity vectors and contours of $u^{\prime}$, b) enlarged view of the region of formation of lower shed vortex showing instantaneous velocity vectors, Event $A$.

The foregoing discussion provides an understanding of some of the basic physics underlying the large variations in shedding period seen in the events investigated here. However, while simple qualitative correlations such as "the presence of high-speed streaks in both the upper and lower boundary layers in a given spanwise region leads to increased shedding frequency in this region" are possible with the understanding obtained thus far, a more quantitative or graphical behavioral model that illustrates the relationship between near-surface boundary-layer velocities encountered at the trailing edge during a high-intensity shedding event, and the observed shedding frequency during the event, is desirable. We now attempt to develop a graphical model for the high-intensity events seen in Fig, 5 (banded and marked with rectangles, not limited to 'simple' events).

Figure 22 is a plot of the event-averaged shedding frequency on the $\mathrm{x}$-axis (normalized by the shedding frequency for all of Case $F$ ) and the event-time-averaged near-surface boundary-layer streamwise velocity $U_{E A}$ on the $y$-axis (normalized by the time-averaged boundary-layer streamwise velocity $U_{A V G}$ for Case $F$ ). Both $U_{E A}$ and $U_{A V G}$ are obtained at $x / D=0.0$. In order that $U_{E A}$ reflects the accelerating/decelerating tendencies of both the upper and lower separated boundary layers, it is defined as $U_{E A}=\left(U_{E A, L B}+U_{E A, U B}\right) / 2$ where $U_{E A, L B}$ and $U_{E A, U B}$ are the event-time-averaged velocities in the lower and upper boundary layers, respectively. The ratio of the velocities plotted on the $y$-axis is a measure of the overall average under-speed/over-speed encountered during an event. The ratio of frequencies on the $x$-axis is a measure of the resulting decrease/increase in shedding frequency. The data plotted in Fig. 22 are for the high-intensity events marked with rectangles in Fig. 5. The near-surface velocities $U_{E A, L B}$ and $U_{E A, U B}$ were obtained at $y / D=-0.75 \& 0.75$, respectively $\left(U_{A V G}\right.$ is also obtained at the same $y$ locations, by 
definition it is the same at both locations). The plot for velocity data obtained closer to the plate surface, at $y / D=-0.65 \& 0.65$, is very similar to that of Fig. 22. Although Fig. 22 shows some scatter in the data, the trend is clear; the higher the average near-surface velocity during an event the larger the resulting eventaveraged shedding frequency. Of importance is that Fig. 22 includes both 'simple' events such as E \& F, and more complex events such as Event $A$; in other words the figure serves to characterize the phenomenon of differences in shedding frequency from event to event caused by high/low speed streaks in the separating boundary layers.

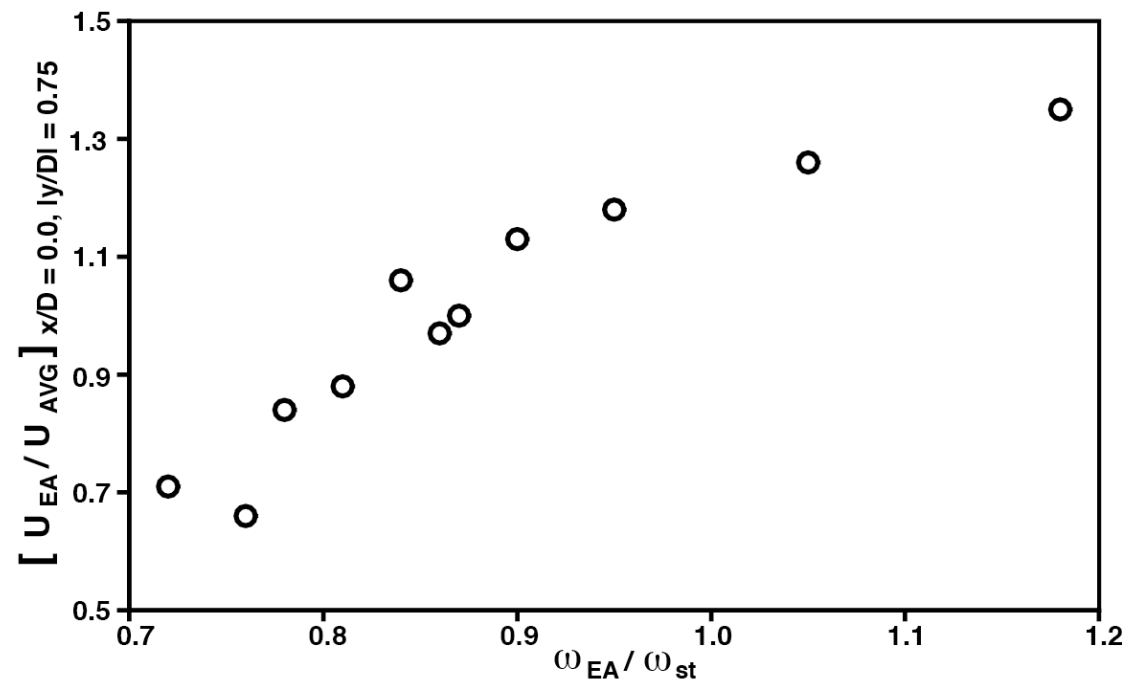

Figure 22. The effect of near-surface boundary-layer under-speed/over-speed on shedding frequency.

It was noted earlier that the strengthening/weakening of shed vortices by streamwise vortices in the separated boundary layer also had implications with regard to shed vortex structure in Case $F$. The shed vortices are enhanced/weakened over the short distances in the $z$ direction over which the influence of the streamwise vortices is felt. Thus this phenomenon is at the same time also a contributor to the finite extent of the bands seen in Fig. 5. The fact that high/low speed streaks in the separating boundary layers increase/decrease shedding frequency implies that these streaks affect shed vortex structure; increases/decreases in shedding frequency are obtained over a z-extent that corresponds to the characteristic widths of these streaks, thus contributing to the finite width of the bands in the z-direction.

\subsection{Vortex shedding in Case G}

Case $\mathrm{G}(\psi=51.59)$ was computed primarily to better understand the effect of a continued increase in $\theta / D$ on shedding characteristics. Figure 23 shows contours of $v$ (as in Fig. 5) over 20 shedding periods for Case $G$ at $x / D=1.0, y / D=0.0$. The spanwise extent shown in the figure is $16 D$. The minimum and maximum values specified and the number of contour levels is the same as in Fig. 5. A comparison of the two figures provides the following observations: a) The vertical bands corresponding to relatively high-intensity shedding are present in Case $G$ as well. b) The bands are not as well structured and are fewer in number within a given time-period and spanwise extent. c) They are longer in the z-direction in Fig. 23 (in terms of the number of diameters) because $D$ is smaller in Case $G$ than in $F$, whereas the band-width is determined by boundary layer streamwise-vortex placement (mean and time-varying boundary layer characteristics are nearly the same in the two cases). d) In general there are fewer bands in each event in Case G. These effects are as expected because the boundary layer disturbance characteristics affecting shedding intensity are nearly the same in the two cases whereas shedding itself is weaker in Case $G$ because of the thinner plate used in this case. It remains to be seen if this increased degradation of the shedding process with increasing $\theta / D$ is the path to an essentially non-shedding state.

Figure 24 shows surfaces of instantaneous constant $\lambda_{2}$ (negative) in the region $0.0<x-D / 2<$ 80.0, $-1.0<y / D<1.0$, and the entire spanwise computational extent (80.0D) for Case G. Sequences of 
approximately spanwise vortices are evident. Some of the sequences are marked with arrows. While one of the marked sequences show vortices well aligned with the $z$ direction, two occur at a significant angle to this direction. Some of the vortices are approximately aligned with the $\mathrm{x}$ direction. As in Case $\mathrm{F}$ (Rai 2017), in the trailing edge region, many of them are nearly streamwise vortices from the upstream boundary layers. Also as in Case F, the spanwise vortices are of finite length in the $z$ direction and the sequences in time also seem to be finite.

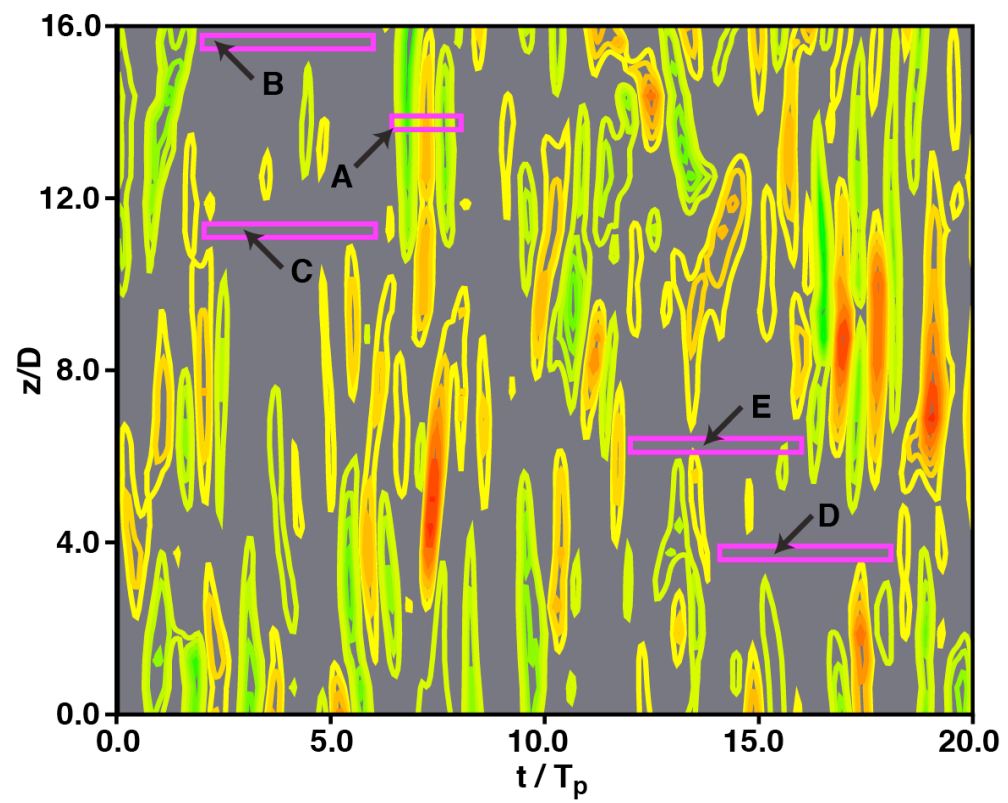

Figure 23. Contours of $v$ at $\mathrm{x} / \mathrm{D}=1.0, \mathrm{y} / \mathrm{D}=0.0$ over 20 shedding periods, Case $\mathrm{G}$.

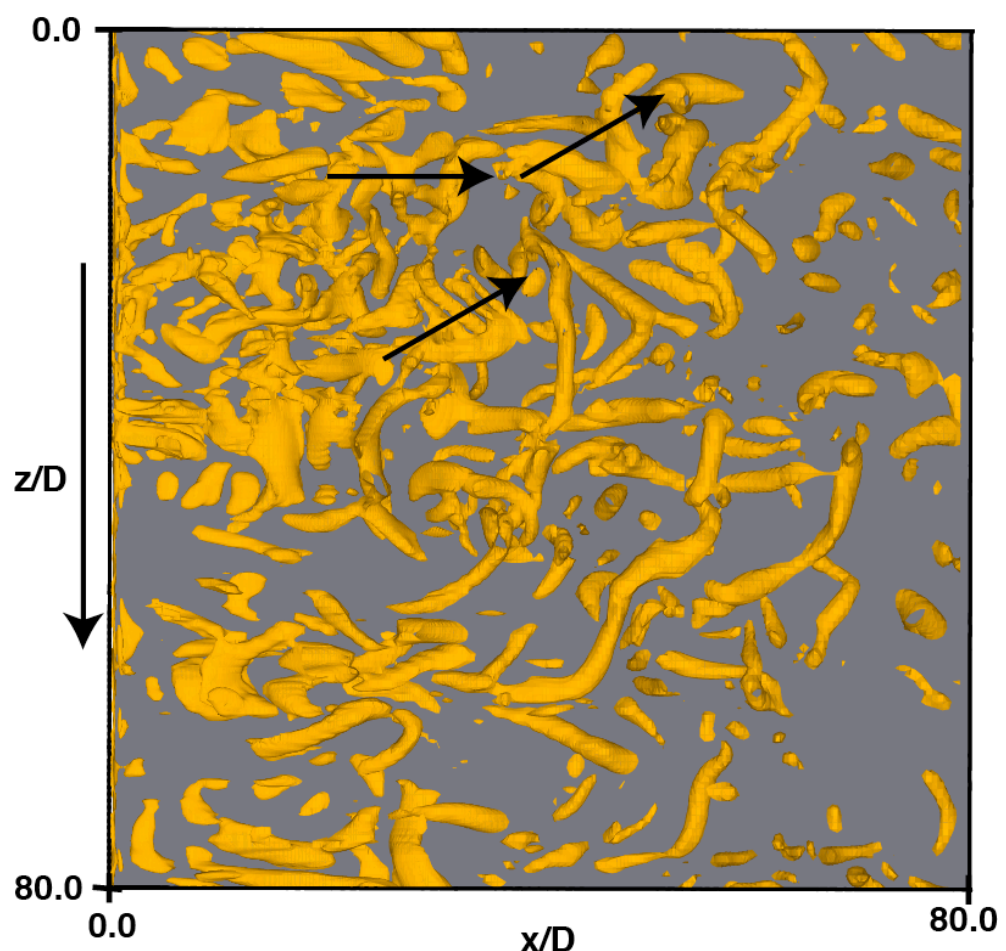

Figure 24. Surfaces of constant $\lambda_{2}$ (negative) in the region $-1.0<y / D<1.0$, Case $G$, (top view). 
One relatively high-intensity shedding event (marked A) and four regions of low-intensity fluctuations (marked B, C, D \& E) are shown in Fig. 23. As expected, an animation of instantaneous velocity vectors in a $(x, y)$ plane during the Event $A$ (z-location of the plane corresponds to the center of the rectangle marked $A$ in Fig. 23), clearly showed the continual formation of the circulating flow associated with the shed vortices. Of interest is the continuity of the formation of regions of circulating flow during the relatively quiescent periods (such as B, C, D \& E). Animations of velocity vectors during each of these events showed the continuity of the process; just as in Case $F$, there was no intermittency (continuity of this process in Case $F$ is discussed in Rai 2017). Figure 25a, for example, shows velocity vectors in $\mathrm{a}(\mathrm{x}, \mathrm{y})$ plane at one instant in time during Event $\mathrm{B}$ (at 1.33 shedding periods past the initiation of this event), which is one of the quiescent periods. The upper region of circulating flow is clearly observed. Figure $25 \mathrm{~b}$ shows the appearance of the subsequent lower region of circulating flow.

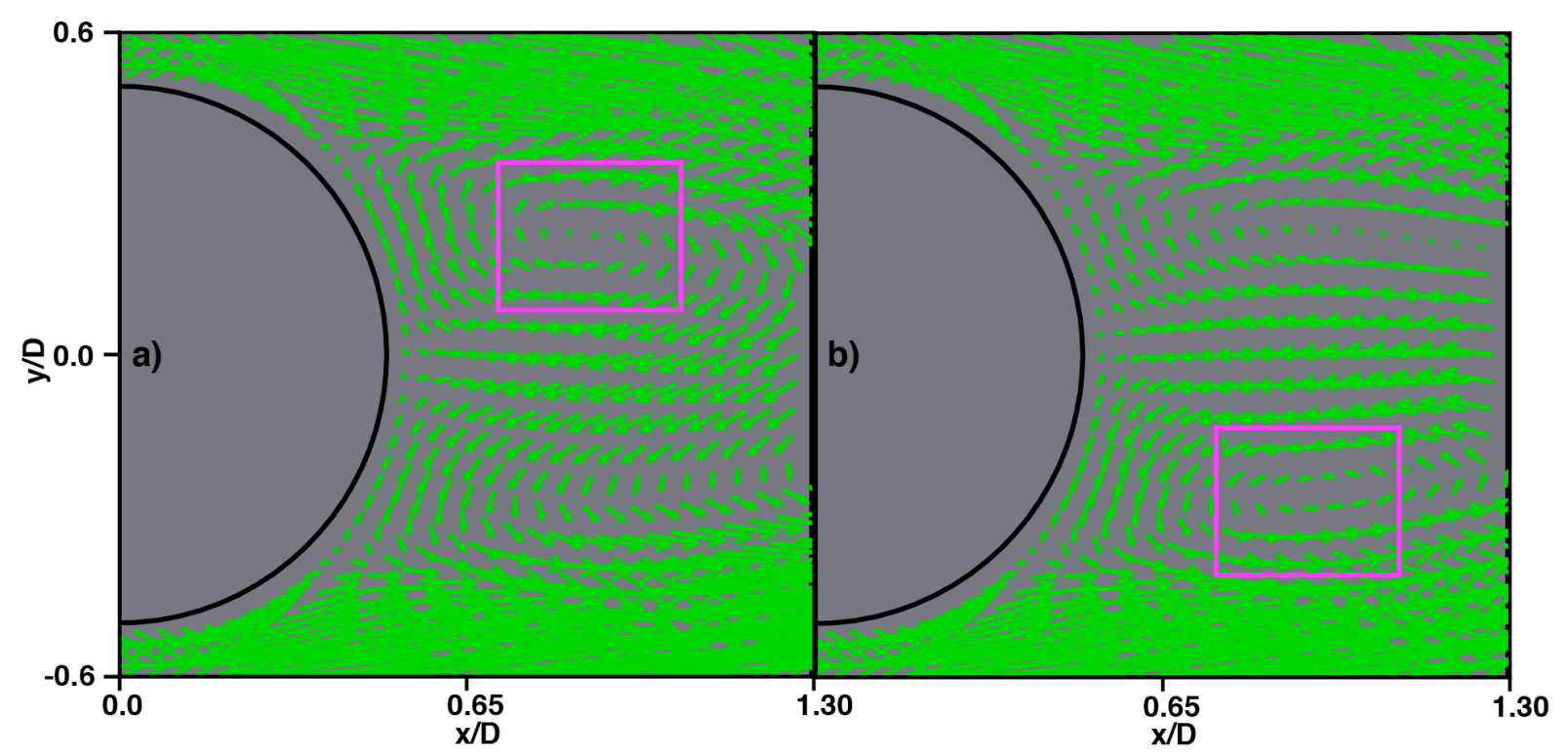

Figure 25. Instantaneous in-plane velocity vectors obtained at two instants in time in Case G, Event B.

The variations in shedding period in Cases $F$ \& $G$ can be expected to widen the peak associated with shedding in spectra of fluctuating $v$ in the wake center-plane. Figure 26 shows spectra for fluctuating $v$ at $x / D=4.0, y / D=0.0$ for Cases $D, F \& G$. The shed-vortices are well formed in all three cases at the streamwise location $x / D=4.0$. All three spectra show a peak at shedding frequency $\omega_{s t}$, Case $D$ exhibits a sharp peak whereas in Case $F$, as expected, the peak is wide. The peak obtained in Case $G$ is barely perceptible. In the investigation of the instability of the detached shear layer of the cylinder Rai (2010), it was found that the time-variation of the shear-layer vortex generation rate was much less than indicated by the energy spread in the spectrum of the fluctuating velocity signal (obtained near the shear layer). The analysis showed that amplitude modulation (generally increasing amplitude during the first half of a period of instability followed by decreasing amplitude during the second half), could be a significant contributor to the broadening of the peak associated with the shear-layer instability. Since Cases F \& G show amplitude modulation in shedding events, the broadening of the spectrum seen in Fig. 26 in these cases may not be entirely because of variation in shedding frequency but also partly due to amplitude modulation.

While distributions in a $(x, y)$ plane of time-averaged (also averaged in the $z$ direction) velocity components, spanwise vorticity, normal intensities and shear stress have been obtained as a part of this investigation, in the interest of brevity, only the variation of normal intensities along the centerline for Cases D, F \& G are included in this article. Earlier investigations by Rai (2013 \& 2014) have included phase-averaged velocity statistics; this is difficult (for reasons discussed in Rai (2017)) and also of limited utility in Cases F \& G. Here, velocity fluctuations include those due to quasi-periodic shedding and the random component. 


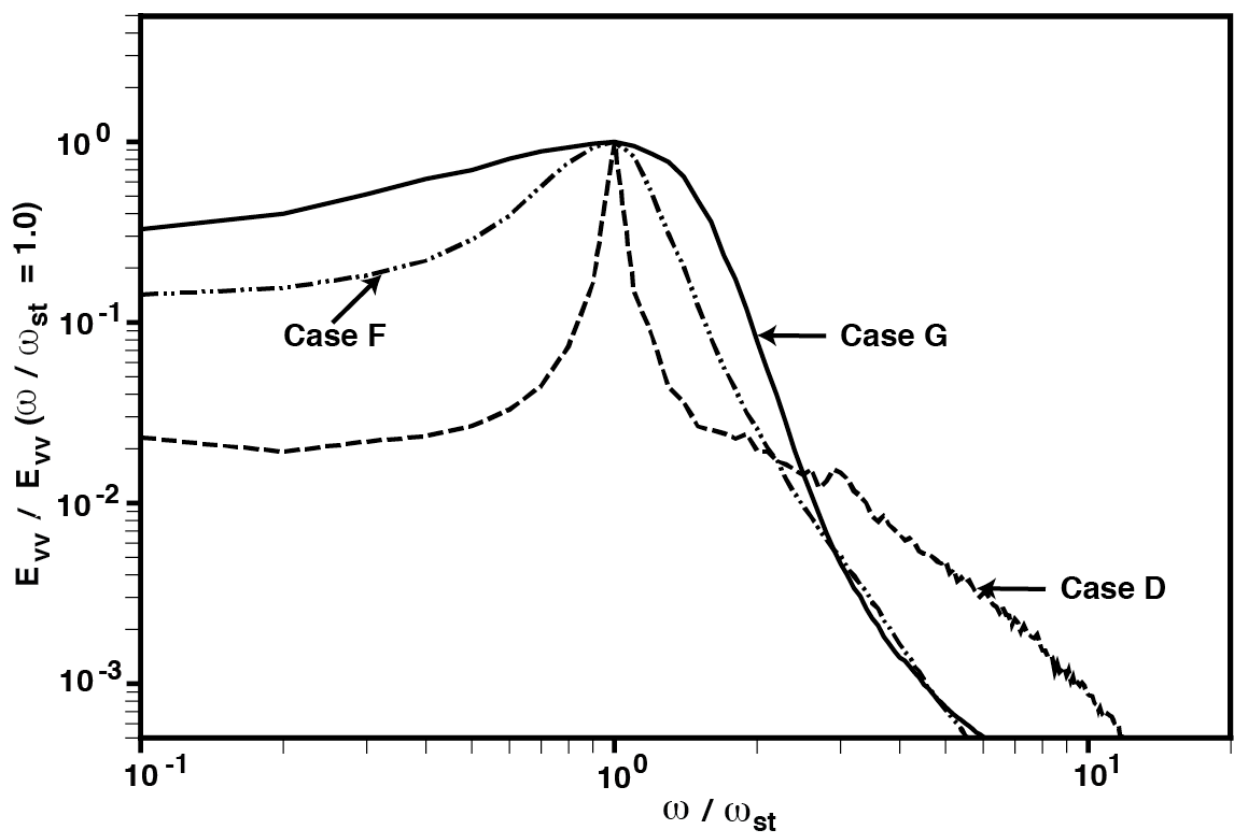

Figure 26. Comparison of spectra obtained for fluctuating $v$ in Cases $D, F$ and $G(x / D=4.0, y / D=0.0)$.

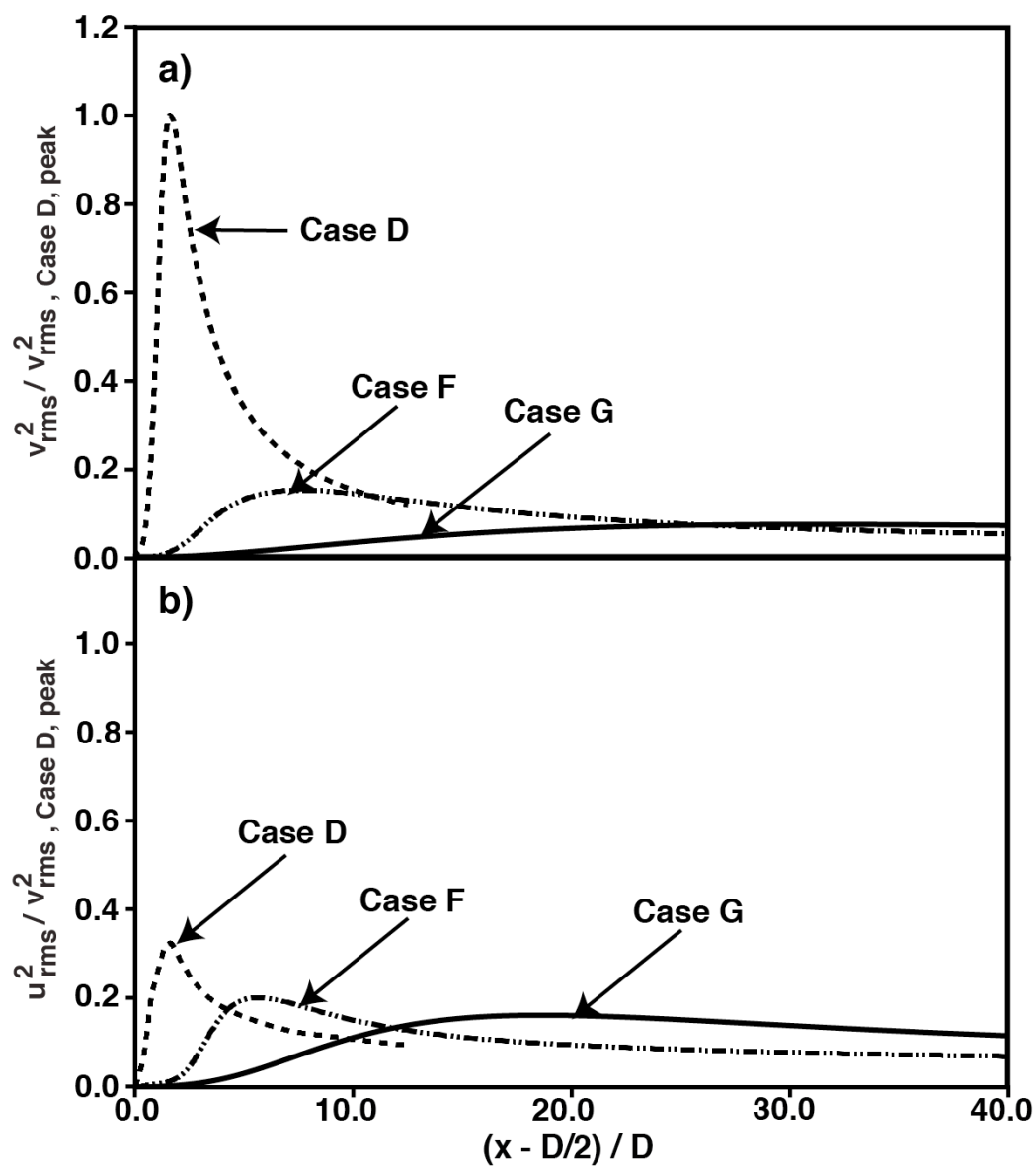

Figure 27. Variation of a) cross-stream and b) streamwise normal intensities along the wake centerline for Cases D, F \& G. 
Figure $27 a$ shows the variation of cross-stream normal intensities along the wake centerline. There is a substantial reduction in peak value and the peak itself occurs further downstream (distance measured in terms of $D$ ) as we move from Case $D$ to Case $G$ (increasing $\theta / D$ ). This is because the shed vortices are weaker and reach their peak strength further downstream as $\theta / D$ is increased. Figure $27 b$ shows the variation of streamwise normal intensities along the wake centerline. While the streamwise intensity level is also diminished with increasing $\theta / D$, much of it is inherited from the boundary layer and therefore is not affected to the same extent. Another feature of interest is that while the peak in crossstream intensity is higher than the peak in streamwise intensity in Case $D$, the reverse is true in Cases $F$ $\& G$. The primary cause for the reversal in trend is again the relative weakness of the shed vortices in Cases $F \& G$ that results in significantly diminished cross-steam intensity levels.

\section{CONCLUDING REMARKS}

The near and very near wake of a thin flat plate with a circular trailing edge are investigated with direct numerical simulations. Data obtained for two different Reynolds numbers (based on plate thickness, D) are the main focus of this study. The separating boundary layers are turbulent in both cases. An earlier investigation of one of the cases (Case F) showed shed vortices in the wake that were about 1.0D to 4.0D in spanwise length. Considerable variation in both the strength and frequency of these shed vortices was observed.

One objective of the present investigation was to determine the important contributors to the variability in the strength of shed vortices. Accordingly the data obtained in Case $F$ was analyzed extensively with the aid of the pertinent terms in the vorticity transport equation for spanwise vorticity. The analysis showed that streamwise vortices in the separated turbulent boundary layer that are in proximity to the shed vortices play a critical role in both strengthening and weakening shed vortices in the wake. The mechanism involves the generation of high levels of the gradient of the spanwise velocity in the $z$ direction $(\partial w / \partial z)$ which then interacts with the spanwise vorticity associated with the detached shear layers to yield regions which either strengthen (positive $\partial w / \partial z$ ) or weaken (negative $\partial w / \partial z$ ) shed vortices. This is quite similar to one of the mechanisms underlying the detached shear layer instability encountered in cylinder and flat plate wakes.

A second objective of the present investigation was to determine the important contributors to the variability in shedding frequency. The contours of cross-stream velocity in a $(t, z)$ plane on points on the wake centerline (fig. 5 in the present article) show that the shedding period (width of pairs of vertical bands) varies from event to event (sometimes even within an event). In some instances the ratio of shedding periods between events is significant; ratios as high as 1.6 were observed between events. A probable cause is the turbulent nature of the separating boundary layer. Fluctuations in the streamwise velocity within the boundary layer could slow down or hasten the convective rate of the shed vortices that are confined to the detached shear layer (as in Cases $F \& G$ ) and thus increase/decrease the shedding period. Here we identified high/low speed streaks, in the turbulent boundary layer near separation, as important contributors to accelerated/decelerated wake vortex shedding. Shed vortices that are embedded in a high-speed streak convect downstream faster and thus result in higher shedding frequencies; low-speed streaks have the opposite effect on the shed vortices.

The strengthening/weakening of shed vortices by streamwise vortices in the separated boundary layer also has implications with regard to shed vortex structure. The shed vortices are strengthened/weakened over the short distances in the $z$ direction where the influence of the streamwise vortices is felt. Thus boundary layer streamwise vortices are at the same time also contributors to the finite extent of the shed vortices in the spanwise direction. The fact that high/low speed streaks in the separating boundary layers increase/decrease shedding frequency also implies that these streaks affect shed-vortex structure. Increases/decreases in shedding frequency are obtained over a z-extent that corresponds to the widths of these streaks, thus contributing to the finite z-extent of the shed vortices. 
Case $G$ was computed primarily to better understand the effect of a continued increase in $\theta / D$ on shedding characteristics. While in Case G vortex-shedding intensity was weaker (as seen from the variation of cross-stream intensity along the wake centerline), and the peak obtained in the spectrum of center-line fluctuating cross-stream velocity was broader than in Case $F$, in general, the main features of the wake were similar to that obtained in Case F. Most importantly, all of the quiescent periods examined showed continuous shedding in Case $G$ as well (shedding continuity in Case F was demonstrated in Rai 2017). This is important in understanding the path to the wake of the thin plate with a sharp trailing edge where shedding is absent. Although there may yet be surprises in store before this end stage is reached, the data analyzed thus far indicate a gradual decline of shedding intensity. It is unclear if there is a small range of $\theta / D$ values over which shedding rapidly ceases to exist.

In all, seven direct numerical simulations of flat plate wakes with turbulent separating boundary layers have been computed to date. In these computations $D$ and $\theta$ vary over a range of 40.0 and about 5.0, respectively. This provides us an opportunity to develop a functional relationship between the shedding frequency and the independent parameters $R_{\theta}$ and $R e_{D}$, and also test the efficacy of the model. Cases A - F were used in developing this behavioral model and Case $G$ was used to test the model in an extrapolative mode (see Appendix). The model yielded a predicted shedding frequency that was less than $6 \%$ higher than that obtained via DNS. While this is a reasonably accurate prediction and this test establishes the efficacy of the model over the range of Reynolds numbers used in developing the model, the more important point is that the exponents obtained for the model indicate a strong dependence of shedding frequency on $\mathrm{Re}_{\mathrm{D}}$ and a relatively weak dependence on $\mathrm{Re}_{\theta}$.

\section{APPENDIX}

\subsection{A Functional Relationship Between Shedding Frequency and $\operatorname{Re}_{\theta} \& \boldsymbol{R e}_{D}$}

The range of Reynolds numbers $\left(R_{L}\right.$ and $\left.R e_{D}\right)$ covered by Cases $A-G$ is substantial and permits the development of various correlations (between the quantity of interest and these two Reynolds numbers). Cases B \& C are discussed in Rai (2015). The Reynolds number based on plate length for these two cases is 302,500 and the $\mathrm{Re}_{\mathrm{D}}$ values are 5,000 and 10000 , respectively; as in all the other cases the freestream Mach number is 0.2. A power-law relationship between the ratio of shear-layer frequency to shedding frequency and $R_{\theta}$ and $R_{D}$ was developed in Rai (2015) using just the Cases $A-$ D. Cases $A-C$ were used to obtain the necessary exponents and constant. The power-law was then used to predict the frequency ratio for Case D. The predicted and computed (via DNS) frequency ratios were very close, 2.57 and 2.58, respectively. The proximity of these two values was obtained even though the power-law was used in an extrapolative mode to predict the frequency ratio for Case $D$ (Cases $A-D$ are at the vertices of a rectangle in $R e_{L}-R e_{D}$ space).

A second power-law relationship between the normalized, phase-averaged, peak spanwise vorticity at shed vortex cores when they are located at $x / D=5.5$ was also developed in Rai (2015) using Cases A - C. The estimated and computed spanwise vorticity values for Case D differed by about $10 \%$. Once again, the power-law was used in an extrapolative mode. Although the estimate is not as accurate as that obtained for the frequency ratio, it is reasonable.

Here we test a functional relationship between the shedding frequency $(\omega)$ and $R e_{\theta}$ and $R e_{D}$. $A n$ initial interrogation of the data revealed a relatively weak dependence of $\omega$ on $\operatorname{Re}_{\theta}(\omega$ decreased with increasing $\mathrm{Re}_{\theta}$ at constant $\mathrm{Re}_{\mathrm{D}}$ ). In addition, Cases $\mathrm{A}, \mathrm{D}, \mathrm{E} \& \mathrm{~F}$ (all with the same plate length) showed only a relatively small variation of $\mathrm{Re}_{\theta}$. Hence, under the assumption that the effect of $\operatorname{Re}_{\theta}$ was very small in these four cases, the variation of $\omega_{A} / \omega$ with $R_{D} /\left(R_{D}\right)_{A}$ was analyzed (the subscript $A$ refers to Case $A$, the reference case). The analysis showed that the relationship between $\omega_{A} / \omega$ and $\operatorname{Re}_{D} /\left(\operatorname{Re}_{D}\right)_{A}$ was close 
to linear. Assuming a power law relationship between $\omega_{A} / \omega$ and $\operatorname{Re}_{\theta} /\left(\mathrm{Re}_{\theta}\right)_{A}$ the following relationship was obtained:

$$
\left(\omega_{A} / \omega\right)=\left(\operatorname{Re}_{\theta} /\left(\operatorname{Re}_{\theta}\right)_{A}\right)^{\alpha}\left(\mathrm{A}+\mathrm{B}\left(\operatorname{Re}_{\mathrm{D}} /\left(\operatorname{Re}_{\mathrm{D}}\right)_{A}\right)^{\beta}\right)
$$

In Eq. $3, A$ and $B$ are constants (with the constraint $A+B=1$ ), and $\operatorname{Re}_{\theta}$ is computed at $x / D=0.0$ (very close to the location of boundary layer separation). Alternatively, Eq. 3 can be written as

$$
\left(\left(\omega_{A} / \omega\right)\left(\left(\operatorname{Re}_{\theta}\right)_{A} / \operatorname{Re}_{\theta}\right)^{\alpha}-\mathrm{A}\right) / \mathrm{B}=\left(\operatorname{Re}_{\mathrm{D}} /\left(\operatorname{Re}_{\mathrm{D}}\right)_{A}\right)^{\beta}
$$

The constants $A$ and $B$ and the exponents $\alpha$ and $\beta$ were obtained from a least squares fit to the computed data for Cases A - F (Case G was not used to obtain the fit but was used instead to test the predictive capability of the model). The values of $\alpha$ and $\beta$ thus obtained are 0.1156 and 1.1520 , respectively. The value of $\alpha$ is nearly an order of magnitude smaller than that of $\beta$. Clearly, variations in $R e_{D}$ result in more significant changes in $\omega$ than similar variations in $R e_{\theta}$. As expected, the value of $\beta$ is close to unity thus showing a nearly linear relationship between $\omega_{A} / \omega$ and $R e_{D} /\left(\operatorname{Re}_{D}\right)_{A}$ and, $\alpha$ is positive thus reflecting the observed trend in the data (increases in $\operatorname{Re}_{\theta}$ result in decreases in $\omega$ at constant $\operatorname{Re}_{D}$ ).

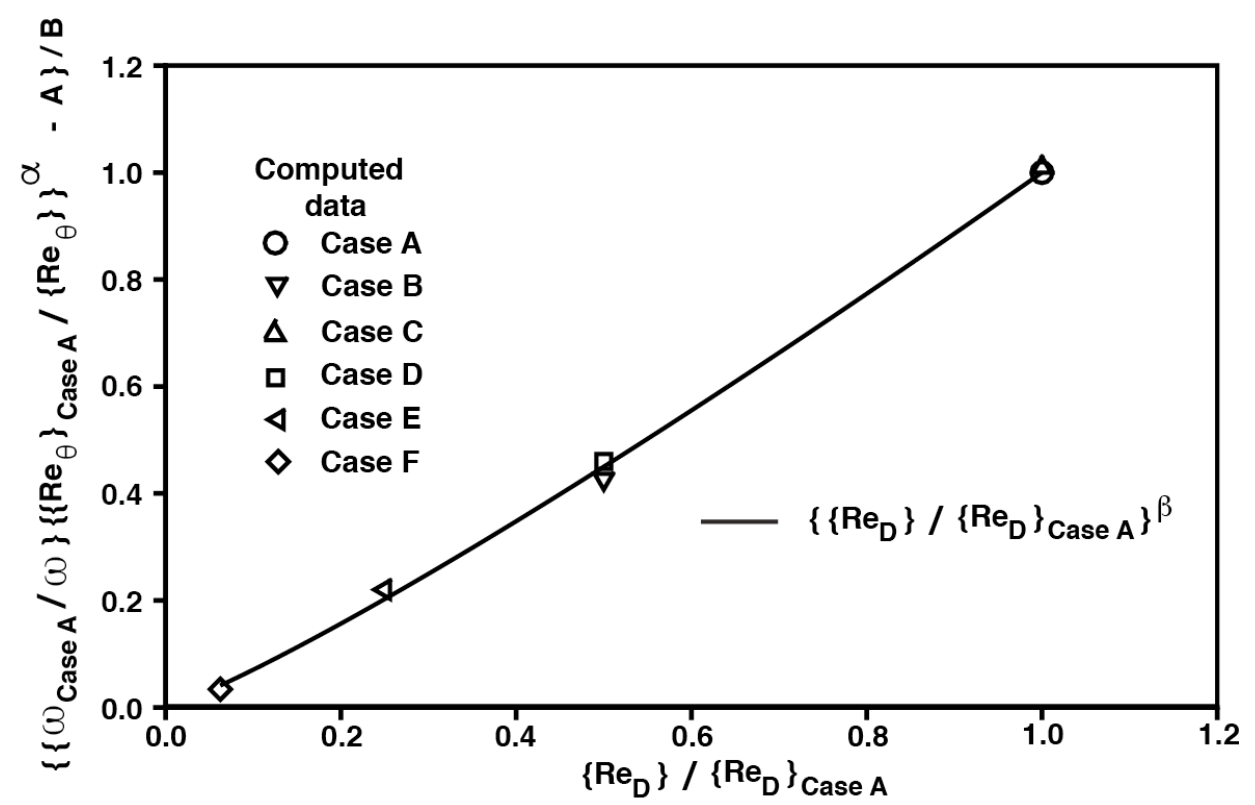

Figure 28. A test of the proposed model relating $\omega$ and $\operatorname{Re}_{\theta} \& \mathrm{Re}_{\mathrm{D}}$ (Eq. 5), using the DNS data and estimated constants/exponents.

Figure 28 shows a plot of the variable

$$
\left(\left(\omega_{A} / \omega\right)\left(\left(\operatorname{Re}_{\theta}\right)_{A} / \operatorname{Re}_{\theta}\right)^{\alpha}-\mathrm{A}\right) / \mathrm{B}
$$

(LHS of Eq. 4) for each of the six cases (values of $\omega$ and $\mathrm{Re}_{\theta}$ obtained from the direct numerical simulations) as a function of $\left(\operatorname{Re}_{D} /\left(\operatorname{Re}_{D}\right)_{A}\right)$. Figure 28 also shows a plot of the function $\left(\operatorname{Re}_{D} /\left(\operatorname{Re}_{D}\right)_{A}\right)^{\beta}$. The computed data (symbols) and the curve are close, thus indicating the efficacy of the model of Eq. 4 (and the computed constants and exponents) for the range of Reynolds numbers used here (a factor of 16 in $\operatorname{Re}_{D}$ and about 5 in $\operatorname{Re}_{\theta}$ for Cases $A-F$ ). The predicted shedding frequency for Case $G$ is about $6 \%$ higher than the computed value. The model is used in an extrapolative mode to obtain this frequency. 


\section{REFERENCES}

ALBER, I. E. 1980 Turbulent wake of a thin, flat plate. AIAA Journal, Vol. 18 (9), 1044.

CHEVRAY, R. \& KOVASZNAY, L. S. G. 1969 Turbulence measurements in the wake of a thin flat plate. AIAA Journal, Vol. 7, 1641.

HAYAKAWA, M. \& lida, S. -I. 1992 Behavior of turbulence in the near wake of a flat plate at low Reynolds number. Physics of Fluids A, Vol. 4 (10) 2282.

JEONG, J. \& HUSSAIN, F. 1995 On the identification of a vortex. Journal of Fluid Mechanics, Vol. 285, 69.

KARLSON, R. I. \& JOHANSSON, T. G. 1988 LDV Measurements of higher-order moments of velocity fluctuations in a turbulent boundary layer. Laser Anemometry in Fluid Mechanics, Ladoan-Instituto Superior Tecnico, Portugal, 273.

KIM, J., MOIN, P. \& MOSER, R. 1987 Turbulence statistics in fully developed channel flow at low Reynolds number. Journal of Fluid Mechanics, Vol. 177, 133.

ONG, L., WALLACE, J., \& MOIN, P. 1995 The velocity and vorticity fields of the turbulent near wake of a circular cylinder. NASA TM -110513.

NAKAYAMA, A. \& LIU, B. 1990 The turbulent near wake of a flat plate at low Reynolds number. Journal of Fluid Mechanics, Vol. 217, 93.

RAI, M. M., \& MOIN, P. 1991 Direct simulations of turbulent flow using finite-difference schemes. Journal of Computational Physics Vol. 96 (1), 15.

RAI, M. M. 2010 A computational investigation of the instability of the detached shear layers in the wake of a circular cylinder. Journal of Fluid Mechanics, Vol. 659, 375.

RAI, M. M. 2013 Flow physics in the turbulent near wake of a flat plate. Journal of Fluid Mechanics, Vol. 724, 704.

RAI, M. M. 2014 Flow Phenomena in the very near wake of a flat plate with a circular trailing edge. Journal of Fluid Mechanics, Volume 756, 510.

RAI, M. M. 2015 Detached shear-layer instability and entrainment in the wake of a flat plate with turbulent separating boundary layers. Journal of Fluid Mechanics, Vol. 774, 5.

RAI, M. M. 2017 Changes in flat plate wake characteristics obtained with decreasing plate thickness. International Journal of Heat and Fluid Flow, Vol. 68, 13.

RAMAPRIYAN, B. R., PATEL, V. C. \& SASTRY, M. S. 1982 The symmetric turbulent wake of a flat plate. AIAA Journal, Vol. 20 (9), 1228.

THOMAS, F. O., \& LIU, X. 2004 An experimental investigation of symmetric and asymmetric turbulent wake development in pressure gradient. Physics of Fluids, Vol. 16, (5), 1725. 